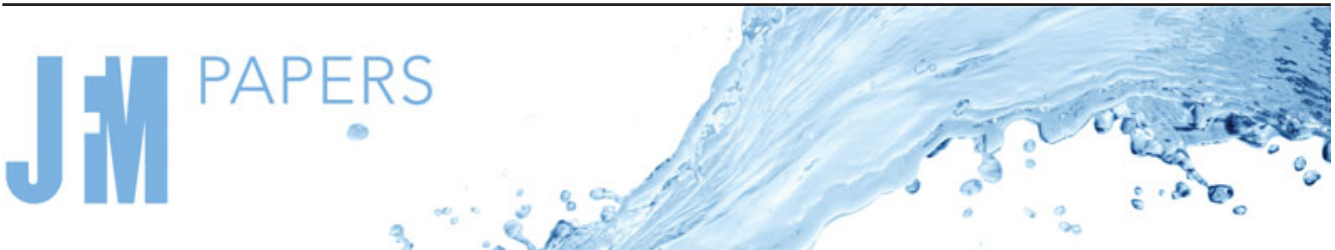

\section{Experimental analysis of the log law at adverse pressure gradient}

\author{
Tobias Knopp ${ }^{1} \dagger$, N. Reuther ${ }^{2}$, M. Novara ${ }^{1}$, D. Schanz ${ }^{1}$, E. Schülein ${ }^{1}$, \\ A. Schröder ${ }^{1}$ and C.J. Kähler ${ }^{2}$ \\ ${ }^{1}$ Institute of Aerodynamics and Flow Technology, German Aerospace Center (DLR), Bunsenstr. 10, \\ 37073 Göttingen, Germany \\ ${ }^{2}$ Institute of Fluid Mechanics and Aerodynamics, Universität der Bundeswehr München (UniBw), \\ Werner-Heisenberg-Weg 39, 85577 Neubiberg, Germany
}

(Received 21 October 2020; revised 18 February 2021; accepted 8 April 2021)

The experimental data for the mean velocity are analysed in the inner layer for a turbulent boundary layer at significant adverse pressure gradient and Reynolds numbers up to $\operatorname{Re}_{\theta}=57000$. The aim is to determine the resilience of the log law for the mean velocity, the possible change of the von Kármán constant $\kappa$ and the appearance of a square-root law above the log law at significant adverse pressure gradients. In the wind-tunnel experiment, the adverse pressure gradient is imposed by an $S$-shaped deflection of the contour model which is mounted on a wind-tunnel sidewall. A large-scale particle imaging velocimetry method is applied to measure the streamwise evolution of the flow over a streamwise distance of 15 boundary layer thicknesses. In the adverse pressure gradient region, microscopic and three-dimensional Lagrangian particle tracking velocimetry are used to measure the mean velocity and the Reynolds stresses down to the viscous sublayer. Oil-film interferometry is used to determine the wall shear stress. The log law in the mean-velocity profile is found to be a robust feature at adverse pressure gradient, but its region is thinner than its zero pressure gradient counterpart, and its slope is altered. A square-root law emerges above the log law, extending to the wall distance the log law typically occupies at zero pressure gradient. Lower values for $\kappa$ are found than for zero pressure gradient turbulent boundary layers, but the reduction is within the uncertainty of the measurement.

Key words: turbulent boundary layers

$†$ Email address for correspondence: tobias.knopp@dlr.de

(C) The Author(s), 2021. Published by Cambridge University Press. This is an Open Access article, distributed under the terms of the Creative Commons Attribution licence (http://creativecommons.org/ licenses/by/4.0/), which permits unrestricted re-use, distribution, and reproduction in any medium, provided the original work is properly cited. 


\section{T. Knopp and others}

\section{Introduction}

Turbulent boundary layers subjected to an adverse pressure gradient still pose many open questions. At the same time, they have a high relevance in many technical applications, e.g. airplane wings, turbomachinery blades and wind turbine blades. Here, the flows around airplane wings are special due to the very high Reynolds numbers $(R e)$. The proper mathematical description of the statistically averaged mean flow of a turbulent boundary layer at a significant adverse pressure gradient (APG) is still under debate. For boundary layers at zero pressure gradient (ZPG), there is large experimental support and agreement in the literature that, for sufficiently large Reynolds numbers, the mean velocity in a large part of the inner layer can be described by the log law

$$
u^{+}=\frac{1}{\kappa} \log \left(y^{+}\right)+B
$$

see e.g. Marusic et al. (2013). The superscript + denotes viscous units. For the von Kármán constant $\kappa$ and for the intercept $B$, Österlund et al. (2000) found $\kappa=0.384$ and $B=4.17$, compared to $\kappa=0.41$ and $B=5.0$ by Coles \& Hirst (1969).

The results for the structure of the mean-velocity profile for turbulent boundary layers at ZPG are supposed to give guidance for the APG case. Traditionally, the boundary layer is divided into four layers, i.e. the viscous sublayer $\left(y^{+}<5\right)$, the buffer layer $\left(5<y^{+}<\right.$ $30)$, the logarithmic layer $\left(30<y^{+}<0.15 \delta^{+}\right)$and the wake layer $\left(y^{+}>0.15 \delta^{+}\right)$, where $\delta$ denotes the boundary layer thickness, see e.g. Wei et al. (2005) for a review. Recent publications indicate agreement on the outer edge of the log layer near $y=0.15 \delta$, see Marusic et al. (2013). The extent of the log law in terms of $\delta$ depends on the method to determine $\delta$. Marusic et al. (2013) determined $\delta$ from a fit of the composite law of the wall/law of the wake, and $\delta$ defined in this way is approximately $25 \%$ to $35 \%$ larger than $\delta_{99}$, see e.g. Marusic et al. (2015). The region $y<0.15 \delta$ will be referred to as the inner layer. Regarding the beginning of the log layer, larger values ranging from $y^{+}>150$ up to $y^{+}>300$ have been proposed recently. A mesolayer located between the buffer layer and the log layer, first proposed by Long \& Chen (1981) and Afzal (1982), was associated with the region $30<y^{+}<300$ in George \& Castillo (1997). Another view was given by Marusic et al. (2013), who found at very high Reynolds numbers the existence of a region where the log law for the mean velocity and a logarithmic profile for the streamwise (and spanwise) turbulence intensities hold simultaneously, leading to $3 \operatorname{Re}_{\tau}^{1 / 2}<y^{+}<0.15 \operatorname{Re}_{\tau}$ for the log-law region. An alternative view was given by Wei et al. (2005) based on a study of the mean momentum balance in differential form. Their log-law region IV is where the mean viscous force loses leading-order influence, and begins at $y^{+}=2.6 R e_{\tau}^{1 / 2}$, see Klewicki, Fife \& Wei (2009).

For flows with a significant APG, the discussion described in Alving \& Fernholz (1995) is still open. A first hypothesis is that the log law (1.1) still holds and that $\kappa$ and $B$ still have the same values as for a turbulent boundary layer at ZPG, but that the region occupied by the log law is progressively reduced with increasing APG. This hypothesis was called the 'progressive breakdown' of the law of the wall in Galbraith, Sjolander \& Head (1977), and was advocated for by, among others, Coles (1956), Perry (1966) and Coles \& Hirst (1969).

In conjunction with the first hypothesis, Perry, Bell \& Joubert (1966) proposed that above the log-law region a so-called half-power-law region arises. In the special case of a vanishing wall shear stress close to separation, the half-power law extends almost down to the wall. This proposal by Stratford (1959) was recently supported by direct numerical 
simulations in Coleman et al. (2017) and Coleman, Rumsey \& Spalart (2018). Modified versions of this wall law are given in Kader \& Yaglom (1978) and in Afzal (2008).

A second hypothesis is that the coefficients $\kappa$ and $B$ of the log law change their values. A functional dependence of $\kappa$ on the pressure gradient parameter in inner scaling $\Delta p_{S}^{+}$

$$
\Delta p_{s}^{+}=\frac{v}{\rho u_{\tau}^{3}} \frac{\mathrm{d} P_{w}}{\mathrm{~d} s}
$$

was proposed by Nickels (2004), where $\mathrm{d} P_{w} / \mathrm{d} s$ denotes the pressure gradient at the wall in the wall-parallel direction, $p$ pressure, $v$ kinematic viscosity and $\rho$ density. An alternative relation was given by Dixit \& Ramesh (2009). To illustrate the extent of variability of $\kappa$, the model by Nickels (2004) predicts a reduction from $\kappa=0.390$ at ZPG to $\kappa=0.370$ for $\Delta p_{s}^{+}=0.01$ at APG. For increasing $\Delta p_{s}^{+}$, the model predicts $\kappa=0.353$ for $\Delta p_{s}^{+}=0.02$, and $\kappa=0.329$ for $\Delta p_{s}^{+}=0.04$. The model by Dixit \& Ramesh (2009) predicts a faster reduction from $\kappa=0.408$ at ZPG to $\kappa=0.376$ for $\Delta p_{s}^{+}=0.01, \kappa=0.346$ for $\Delta p_{s}^{+}=$ 0.02 and $\kappa=0.293$ for $\Delta p_{s}^{+}=0.04$. Therefore, experimental support for the hypothesis of a variability of $\kappa$ is supposed to require values of $\Delta p_{s}^{+}>0.01$. Regarding a possible change of $\kappa$ and $B$, a number of data sets were evaluated by Monkewitz, Chauhan \& Nagib (2008), who found an empirical correlation between $\kappa$ and $B$. Experimental results by Nagano, Tagawa \& Tsuji (1991) could indicate a change in $B$, and the direct numerical simulation (DNS) data by Lee \& Sung (2009) give indications that $\kappa$ and $B$ may change.

A third hypothesis is that the pressure gradient causes ' $[\ldots]$ a change in the character of the velocity distribution over the entire region [...]' occupied by the log law in a ZPG flow, see Galbraith et al. (1977), called a 'general breakdown' of the log law. Some authors proposed a single formulation for the entire inner layer based on the half-power law (or square-root law, abbreviated: sqrt-law), see Townsend (1961)

$$
u^{+}=\frac{1}{K_{o}}\left[\log \left(y^{+}\right)+2\left(\sqrt{1+\Delta p_{s}^{+} y^{+}}-1\right)+2 \log \left(\frac{2}{\sqrt{1+\Delta p_{s}^{+} y^{+}}+1}\right)\right]+B_{o} .
$$

In the theoretical analysis, the half-power law is assumed to be associated with the total shear stress growing linearly with the wall distance, see e.g. Brown \& Joubert (1969).

In this work, the analysis of a new turbulent boundary layer experiment is presented, whose aim was to answer the following questions for the mean velocity profile:

(Q1) Does a log-law region still exist at APG?

(Q2) Does the von Kármán constant $\kappa$ change with $\Delta p_{s}^{+}$, see Nickels (2004)?

(Q3) Is there a sqrt-law region above the log law, see e.g. Perry et al. (1966)?

These questions were motivated by a literature study and by the results of the precursor experiment by Knopp et al. (2014b). Therein, a turbulent boundary layer was studied, where the flow followed an $S$-shaped deflection of the geometry model, which caused a strong APG up to $\Delta p_{s}^{+}=0.06$ and $R e_{\theta}$ up to 18000 , see figure 2 in Knopp et al. (2014a). A three-layer form for the mean velocity as described by Perry et al. (1966) was found, see figure 3 in Knopp et al. (2014a). A small log-law region was indicated from a thin plateau of the slope diagnostic function, see figure 5 in Knopp et al. (2014a), and $\kappa$ was found to be reduced in the APG region, see figure 6 in Knopp et al. (2014b). A half-power law was observed above the log law, albeit only over a small region in terms of $y^{+}$. The question arose as to whether a higher Re leads to a larger extent of the log law and of the half-power-law region. 


\section{T. Knopp and others}

Several new experiments and numerical simulations for turbulent boundary layers at APG have been provided during the last decade. Wind-tunnel experiments were performed by, e.g. Atkinson et al. (2016), Monty, Harun \& Marusic (2011), Harun et al. (2013) and Schatzman \& Thomas (2017). New numerical simulations were accomplished by, e.g. Lee \& Sung (2009), Gungor et al. (2016), Coleman et al. (2017) and Coleman et al. (2018). Regarding the mean flow and the turbulence statistics, most of the work focussed on the outer part of the boundary layer, whereas the inner layer was studied in detail only in Coleman et al. (2017) and in Coleman et al. (2018).

Flow experiments with pressure gradients and streamwise surface curvature are rare in the literature, see Baskaran, Smits \& Joubert (1987) and Bandyopadhyay \& Ahmed (1993). Flows with surface curvature alone have been studied in depth since the work by Bradshaw (1970). For the present work, convex curvature is relevant. The magnitude of curvature effects depends on the ratio of the local boundary layer thickness $\delta$ to the local radius of curvature $R_{c}$. Large curvature effects are associated with values for $\delta / R_{c}>0.05$, see e.g. Gillis \& Johnston (1983). Values of $\delta / R_{c}<0.01$ are associated with mild curvature, which were studied e.g. by Ramaprian \& Shivaprasad (1978). The relaxation of a turbulent boundary layer from curvature on a flat plate was studied for the ZPG case by Gillis \& Johnston (1983) and by Alving, Smits \& Watmuff (1990).

The focus of the present work is on the behaviour of the mean-velocity profile, and we only use the single-point statistics. In complementary publications the simultaneous spatial information provided by the 2D2C data, i.e. planar data (two-dimensional) of two components $(2 \mathrm{C})$ of the velocity, and 3D3C data, i.e. volumetric data for all three components (3C) of the velocity were exploited. The characterisation of coherent structures is described in Reuther et al. (2015) and Reuther (2019). The interaction of coherent flow structures is studied in Bross, Fuchs \& Kähler (2019). Their representation using the attached eddy model is described in Eich et al. (2020), and the intermittent behaviour is analysed in Reuther \& Kähler (2018), Reuther (2019) and Reuther \& Kähler (2020).

This paper is organised as follows. The wind-tunnel experiment is described in $\S 2$. The description of the flow is given in $\S 3$. The central part is formed by the results in the APG region and their analysis in $\S 4$. In $\S 5$, history effects in the inner and outer layers are discussed. The conclusions of the analysis are summarised in $\S 6$.

\section{Experimental investigation}

The aim of the experiment was to answer the three questions formulated in the introduction. Regarding the hypothesis by Nickels (2004), (A7) was used to estimate the supposed change of $\kappa$. This led to the first design condition (C1) to reach $\Delta p_{s}^{+}>0.01$ in the focus region of the APG, so that a possible change of $\kappa$ due to the pressure gradient is large enough to be distinguished from uncertainties related to the evaluation of $\kappa$ and the determination of $u_{\tau}$. For this purpose, the measurements were performed on the contour geometry model and not on the flat wind-tunnel wall opposite to the model, since the values of $\Delta p_{s}^{+}$are significantly larger on the contour model. The second design condition (C2) was to reach large Reynolds numbers in the APG focus region, based on the assumption that only at large Reynolds numbers does the asymptotic structure of the wall law with significantly thick log-law and sqrt-law regions form.

Two additional conditions were a consequence of the aim to use the measurement technique as accurate as possible. The third condition (C3) was to use a flat surface in the APG focus region to enable measurements through a glass plate from behind to reduce the 


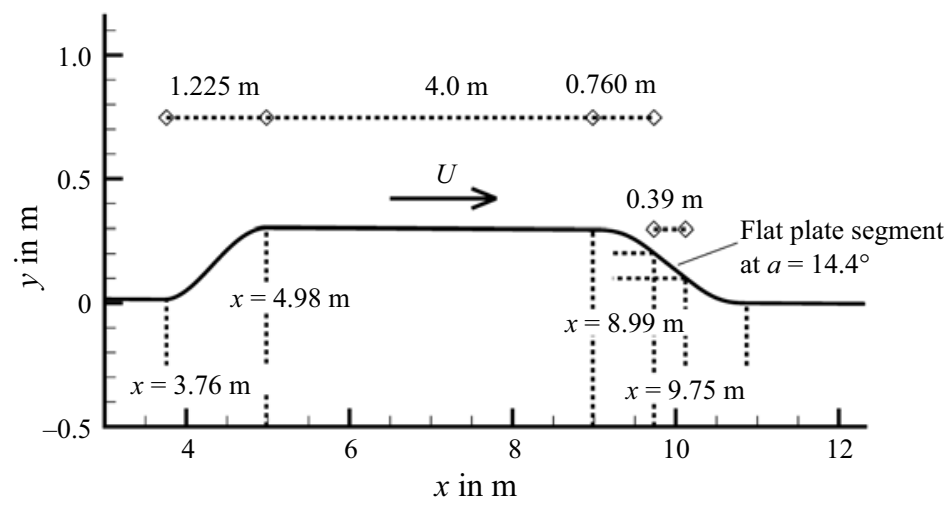

Figure 1. Sketch of the wind-tunnel experiment with flow direction (axes not to scale).

issue of reflections of particle imaging methods in the near-wall region. The fourth design condition (C4) was to achieve large Reynolds numbers at moderately low flow speeds and large boundary layer thicknesses to enable accurate measurements in the viscous sublayer. Due to the design condition (C4) in conjunction with the decision to measure on the geometry model, the issue of surface curvature effects arose. We accepted this issue. The option was to reach $\Delta p_{s}^{+}>0.01$ on the wind-tunnel wall and a much stronger pressure gradient on the geometry model, causing the flow to separate. This idea was abandoned, since it would have meant to either accept a three-dimensional separation or to use flow actuation to prevent separation. The latter was not pursued due to the technical challenges to achieve well-defined and reproducible flow conditions.

\subsection{Design of the experiment and set-up in the wind tunnel}

The experiment was performed in the Eiffel type atmospheric wind tunnel of UniBw in Munich, which has a $22 \mathrm{~m}$ long test section with a rectangular cross-section of $2 \mathrm{~m} \times 2 \mathrm{~m}$. As described in figure 1, the flow develops on the sidewall of the wind tunnel over around $4 \mathrm{~m}$ and is then accelerated along a first ramp of height $0.30 \mathrm{~m}$ and of length $1.225 \mathrm{~m}$. Then, the flow gradually develops along a flat plate of length $4.0 \mathrm{~m}$ with ZPG into an equilibrium. The flow follows a curvilinear deflection of length $l_{c}=0.75 \mathrm{~m}$ which initially causes a small favourable pressure gradient (FPG), and enters into the APG region. The focus region is an inclined flat plate of length $0.4 \mathrm{~m}$, beginning at $x=9.75 \mathrm{~m}$, at an opening angle of $\alpha=14.4^{\circ}$ with respect to the $4.0 \mathrm{~m}$ long flat plate. Finally, the flow follows a second deflection down to the wind-tunnel wall. The opening angle was chosen to keep the flow remote from separation in a more conservative way than in the precursor experiment by Knopp et al. (2014b) and was designed based on computational fluid dynamics (CFD) results with the DLR TAU code using the Spalart-Allmaras model and the shear stress transport (SST) $k-\omega$ model, where $k$ is turbulent kinetic energy and $\omega$ is specific dissipation rate.

The coordinate system shown in figure 1 denotes by $x$ the direction parallel to the floor of the wind tunnel. The origin $x=0$ is defined at the nominal beginning of the test section, which is located $0.875 \mathrm{~m}$ downstream of the thinnest cross-section of the contraction. The curvilinear deflection can be described by a fourth-order polynomial $f(\zeta)$. Here, $\zeta$ denotes the relative coordinate $\zeta=x-8.99 \mathrm{~m}$, i.e. $\zeta=0$ at the beginning of the curvilinear element and $\zeta=l_{c}$ at its end. Then the conditions of a smooth transition between the flat plate and the curved wall imply $f^{\prime}(0)=0, f^{\prime}\left(l_{c}\right)=a=\arctan (\pi \alpha / 180)$ with $\alpha=$ 


\section{T. Knopp and others}

$\begin{array}{lccc} & 2 \mathrm{D} 2 \mathrm{C} \mathrm{PIV} & \text { LR- } \mu \mathrm{PTV} & \text { Three-dimensional LPT (STB) } \\ U_{e} \text { at } x_{r e f} & 28.1 \mathrm{~m} \mathrm{~s}^{-1} & 28.1 \mathrm{~m} \mathrm{~s}^{-1} & 43.2 \mathrm{~m} \mathrm{~s}^{-1} \\ \text { Field of view } & 0.44 \times 0.37 \mathrm{~m}^{2} & 20 \times 17 \mathrm{~mm}^{2} & 50 \times 90 \times 8 \mathrm{~mm}^{3} \\ \text { Resolution } & 5.9 \mathrm{px} \mathrm{mm}^{-1} & 125 \mathrm{px} \mathrm{mm}^{-1} & 35 \mathrm{px} \mathrm{mm}^{-1} \\ \text { Interrogation volume } l_{x} \times l_{y} \times l_{z} & 2.7 \times 2.7 \times 1 \mathrm{~mm}^{3} & - & - \\ \text { IW size } l_{y} / \text { bin size at } x_{r e f} & 91 \delta_{v} & 0.27 \delta_{v} & 2.88 \delta_{v} \\ \delta_{v}=v / u_{\tau} \text { at } x_{r e f} & 30 \mu \mathrm{m} & 30 \mu \mathrm{m} & 20 \mu \mathrm{m}\end{array}$

Table 1. Summary of the experimental parameters for the reference position $x_{r e f}=9.944 \mathrm{~m}$. The flow was seeded with DEHS droplets with a diameter of approx. $1 \mu \mathrm{m} . \mathrm{px}$, pixel.

$14.4^{\circ}$, and $f^{\prime \prime}(0)=f^{\prime \prime}\left(l_{c}\right)=0$, where $f^{\prime}$ and $f^{\prime \prime}$ denote the first and second derivatives. This leads to $f(\zeta)=-a /\left(2 l_{c}^{3}\right) \zeta^{4}+a / l_{c}^{2} \zeta^{3}$. In order to reduce the effects of the sidewalls, the dimension of the APG part of the geometry was reduced by a factor of two compared to the previous experiment by Knopp et al. (2014b).

The experimental results presented here were performed at a free-stream velocity $U_{\infty}=$ $23 \mathrm{~m} \mathrm{~s}^{-1}$ and $U_{\infty}=36 \mathrm{~m} \mathrm{~s}^{-1}$, measured at a reference position near the beginning of the test section. The values for $R e_{\theta}$ of 24400 and 35900 at $x=8.12 \mathrm{~m}$ (ZPG region) respectively 40000 and 57400 at $x=9.944 \mathrm{~m}$ (APG region) are among the highest after the experiments in Coles \& Hirst (1969), and comparable to those of Skare \& Krogstad (1994) and Nagib, Christophorou \& Monkewitz (2004). The static pressure measurements were performed using two DTC Initium Systems, where 64 channels were used in parallel, in the centreline and in different spanwise planes. The free-stream turbulence intensity (FSTI) was quantified in the empty test section in Schulze (2012). The mean FSTI was $0.14 \%$ measured at $x=2.5 \mathrm{~m}$ for $U_{\infty}=38 \mathrm{~m} \mathrm{~s}^{-1}$, averaged over the entire cross-section. The FSTI variation was found to be between $0.10 \%$ and up to $0.19 \%$ towards the corners. The variation of the FSTI was not measured in the flow direction for the wind tunnel with the contour model. Therefore, a possible influence on the boundary layer could not be assessed.

\subsection{Measurement technique}

Different particle imaging approaches were combined in order to measure the mean velocity and the Reynolds stresses over a streamwise extent of several boundary layer thicknesses from the outer edge of the boundary layer down to the viscous sublayer.

\subsubsection{Large-scale $2 D 2 C$ particle image velocimetry}

For an overview measurement from $x=8 \mathrm{~m}$ to $x=10.2 \mathrm{~m}$ a multi-camera large-scale 2D2C-particle image velocimetry (PIV) measurement was applied using 9 cameras, named c1 to c9. The cameras $\mathrm{c} 1$ to $\mathrm{c} 7$ were located in the region of ZPG, FPG and mild APG, whereas the cameras c8 and $\mathrm{c} 9$ were located in the region of the largest APG. The 2D2C-PIV data were evaluated using a single-pixel ensemble correlation and a window correlation method, see Reuther et al. (2015). The interrogation window (IW) size was $16 \mathrm{px} \times 16 \mathrm{px}$ and the interrogation step size was $8 \mathrm{px}$, corresponding to an overlap of $50 \%$. From the 2D2C-PIV data, the mean-velocity profiles were extracted at 13 selected streamwise positions. Details of the PIV method are given in table 1.

The spatial resolution of the PIV method depends mainly on the magnification of the imaging system, the pixel size of the recording cameras and the selected IW dimensions, 
see Kähler, Scharnowski \& Cierpka (2012a). To locally capture the entire boundary layer, the nine sCMOS cameras were equipped with $50 \mathrm{~mm}$ Zeiss lenses (c1-c7) and $35 \mathrm{~mm}$ Zeiss lenses (c8-c9), respectively. In the regions of ZPG, FPG and mild APG, the field of view was $0.32 \mathrm{~m} \times 0.27 \mathrm{~m}$ and the interrogation volume size was $2 \mathrm{~mm} \times 2 \mathrm{~mm} \times 1 \mathrm{~mm}$

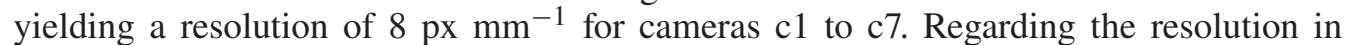
viscous units $\delta_{v}$ at $x=8.12 \mathrm{~m}$, the IW size was $l_{y}^{+}=125$ based on $\delta_{v}=v / u_{\tau}=16 \mu \mathrm{m}$ for $U_{\infty}=23 \mathrm{~m} \mathrm{~s}^{-1}$. In the APG region the field of view was $0.44 \mathrm{~m} \times 0.37 \mathrm{~m}$ and the interrogation volume $l_{x} \times l_{y} \times l_{z}$ was $2.7 \mathrm{~mm} \times 2.7 \times 1 \mathrm{~mm}$. The IW size in the wall-normal direction was $l_{y}^{+}=91$ for camera c8 at $x_{\text {ref }}=9.944 \mathrm{~m}$.

\subsubsection{Particle tracking velocimetry}

To resolve the near-wall region, a high magnification approach using long-range microscopic particle tracking velocimetry (2D- $\mu$ PTV), see Kähler, Scharnowski \& Cierpka $(2012 b)$, was applied at the position $x_{r e f}=9.944 \mathrm{~m}$ in the APG region. The wall-normal extent of the field of view was $140 \delta_{v}$ for the case $U_{\infty}=23 \mathrm{~m} \mathrm{~s}^{-1}$. The size of a bin in the wall-normal direction was $0.27 \delta_{v}$.

The three-dimensional Lagrangian particle tracking (LPT) approach using the shake-the-box (STB) method was used for the case $U_{\infty}=36 \mathrm{~m} \mathrm{~s}^{-1}$, see Novara et al. (2016). The macroscopic field of view of $50 \mathrm{~mm} \times 90 \mathrm{~mm} \times 8$ covered approximately $0.4 \delta_{99}$. In this work we use the data which were sampled over a bin size of $2.88 \delta_{v}$ in the wall-normal direction. This evaluation will be referred to as LPT detail.

For a study of the terms of the mean momentum equation, a second evaluation was performed. The field of view was divided into 111 bins in the wall-normal direction and 5 bins in the wall-parallel direction, corresponding to a bin size of $321 \mathrm{px} \times 30 \mathrm{px}$ (or $9.2 \mathrm{~mm} \times 0.86 \mathrm{~mm}$ ) in the streamwise and wall-normal directions. The gradients were evaluated using a linear interpolation over a kernel of 5 points located in the centre of each bin. The choice of the large bin size in streamwise direction was motivated by statistical convergence reasons, as more than 400000 entries per bin are available to estimate the mean and fluctuating velocity components. Since the bin size in wall-normal direction corresponds to around $41 \delta_{v}$, this evaluation is referred to as LPT average (abbreviated LPT ave).

\subsubsection{Oil-film interferometry}

The wall shear stress was measured using oil-film interferometry (OFI) from $x=8.33 \mathrm{~m}$ to $x=10.02 \mathrm{~m}$. This provides absolute measurements of the wall shear stress independent of any assumption on the mean-velocity profile. The uncertainty of the OFI measurement technique for determining the average friction velocity was estimated to be smaller than $2 \%$ (with a $95 \%$ confidence level) based on Thibault \& Poitras (2017). For technical details of the oil-film interferometry measurements we refer to Schülein, Reuther \& Knopp (2017). The experiments using the different measurement techniques were performed at different days, nominally for the same values of $U_{\infty}$, see table 8 in appendix B. It was not possible to repeat the OFI measurements to obtain the same Reynolds numbers, since the contour model was deployed shortly after the measurement campaign.

\section{Description of the flow}

The flow and its streamwise evolution are described using the overview measurement. The aim is to show that the experimental set-up provides the intended flow conditions in 


\section{T. Knopp and others}
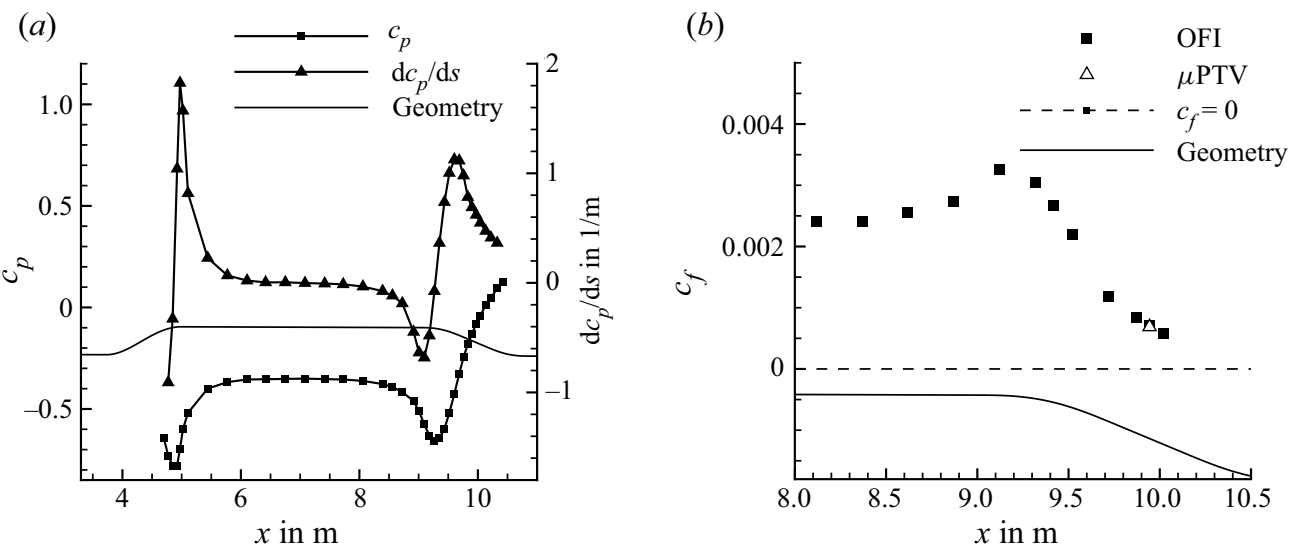

Figure 2. Streamwise distribution of $c_{p}$ and $\mathrm{d} c_{p} / \mathrm{d} s(a)$ and $c_{f}$ from OFI $(b)$ for $U_{\infty}=23 \mathrm{~m} \mathrm{~s}^{-1}$.

the APG region. The set-up leads to a streamwise changing pressure gradient and convex surface curvature effects, which are also described.

\subsection{Streamwise evolution of boundary layer parameters}

The streamwise distribution of the pressure coefficient $c_{p}$ is shown in figure $2(a)$. Moreover, $\mathrm{d} c_{p} / \mathrm{d} s$ is shown, where $s$ is the coordinate direction tangential to the contour wall. Note that $U_{r e f}=U_{\infty}$ was used for the non-dimensionalisation of $c_{p}=(p-$ $\left.p_{r e f}\right) / q_{r e f}$, with $q_{r e f}=\rho_{r e f} U_{r e f}^{2} / 2$. The streamwise distribution of $c_{f}=\tau_{w} / q_{r e f}$ from OFI is shown in figure $2(b)$ for $U_{\infty}=23 \mathrm{~m} \mathrm{~s}^{-1}$. Therein, $U_{r e f}=U_{e}$ at $x=8.12 \mathrm{~m}$ is used for non-dimensionalisation. In the focus region at $x=9.944 \mathrm{~m}$ the flow is not close to separation. Downstream of $x=10.0 \mathrm{~m}$, the flow remains attached with $c_{f}$ significantly larger than zero, as inferred from the 2D2C PIV data. There were no indications for corner flow separation in the junction of the contour model and the wind-tunnel sidewall from tuft flow visualisation.

The boundary layer thickness was evaluated using different methods, see figure 3(a). The conventional definition $\delta_{99}$ and $\delta_{995}$ for a flat plate at ZPG uses the wall distance where the wall-parallel velocity reaches $99 \%$ and $99.5 \%$ of its maximum value along a wall-normal line. The maximum value could be determined for all profiles in the region $8 \mathrm{~m}<x<10.2 \mathrm{~m}$. On the curved wall, the profiles show a distinct maximum. On the flat wall in the APG region, $\partial U / \partial y$ becomes zero when approaching the boundary layer edge, see figure $3(b)$. Appendix $\mathrm{C}$ describes the other criteria used based on the generalised velocity $\tilde{U}$ by Coleman et al. (2018), which is shown in figure $3(b)$, on $u^{\prime} / U$ by Vinuesa et al. (2016), on the potential velocity $U_{p}$ for curved walls by Patel \& Sotiropoulos (1997) and on the turbulent/non-turbulent interface (TNTI) by Reuther \& Kähler (2018). The values for $\delta_{99}$ are found to be in close agreement with the different proposals, and are therefore used to describe the boundary layer thickness in the following.

The pressure gradient along the contour model is shown in terms of $\Delta p_{s}^{+}$in figure $4(a)$ and the Clauser-Rotta scaling $\beta_{R C}=\delta^{*} /\left(\rho u_{\tau}^{2}\right) \mathrm{d} P_{w} / \mathrm{d} s$ in figure $4(b)$. Both become large in the APG region where $u_{\tau}$ becomes small. The pressure gradient parameter $\beta_{Z S}=$ $\delta_{99}^{2} /\left(\rho U_{e}^{2} \delta^{*}\right) \mathrm{d} P_{w} / \mathrm{d} s$ in the scaling by Zagarola \& Smits (1997) and Gungor et al. (2016), which does not involve $u_{\tau}$, is shown in figure $4(c)$. Downstream of the ZPG region, $\beta_{Z S}$ shows significant negative values for the FPG near $x=9.05 \mathrm{~m}$, then changes its sign and 

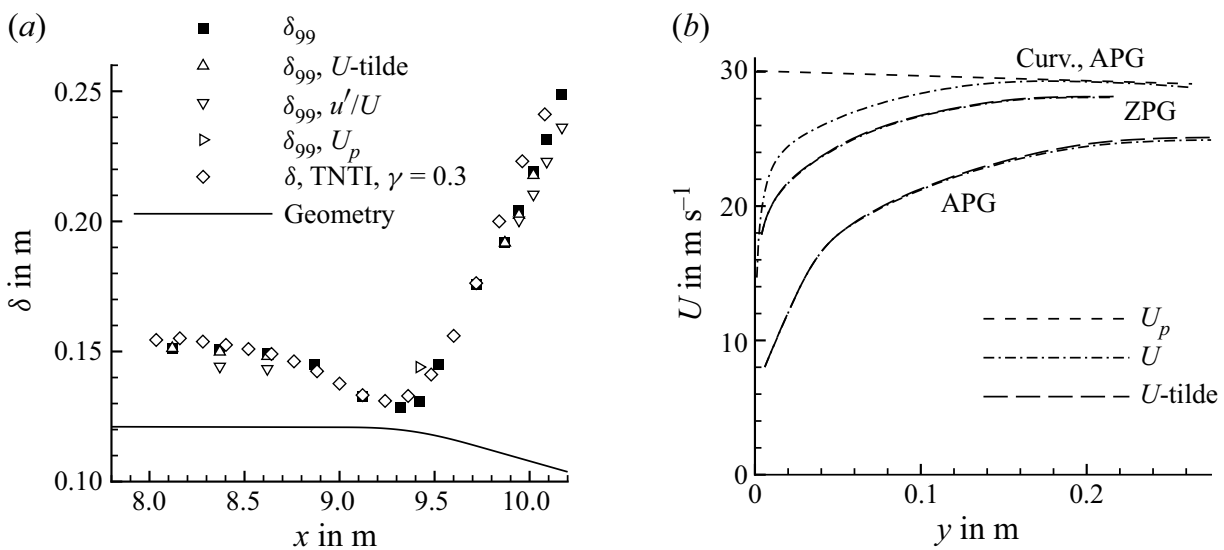

Figure 3. (a) Streamwise distribution of the boundary layer thickness evaluated using different criteria: U-tilde: $\tilde{U}$ by Coleman et al. (2018); $u^{\prime} / U$ : by Vinuesa et al. (2016); $U_{p}$ : by Patel \& Sotiropoulos (1997); TNTI, $\gamma=0.3$ : by Reuther \& Kähler (2018). (b) Mean velocity profile for $U, \tilde{U}$ and $U_{p}$ at the ZPG position $x=8.12 \mathrm{~m}$, at $x=9.42 \mathrm{~m}$ on the curved wall at APG, and at $10.02 \mathrm{~m}$ on the inclined flat wall element at APG for $U_{\infty}=23 \mathrm{~m} \mathrm{~s}^{-1}$.
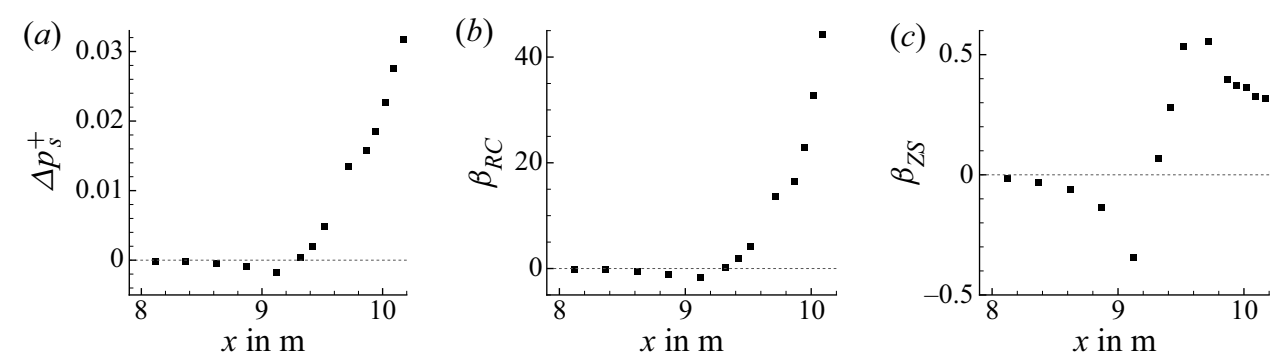

Figure 4. Streamwise pressure gradient parameter $(a)$ in inner scaling, $(b)$ in Clauser-Rotta scaling and $(c)$ in the scaling by Zagarola \& Smits for $U_{\infty}=23 \mathrm{~m} \mathrm{~s}^{-1}$.
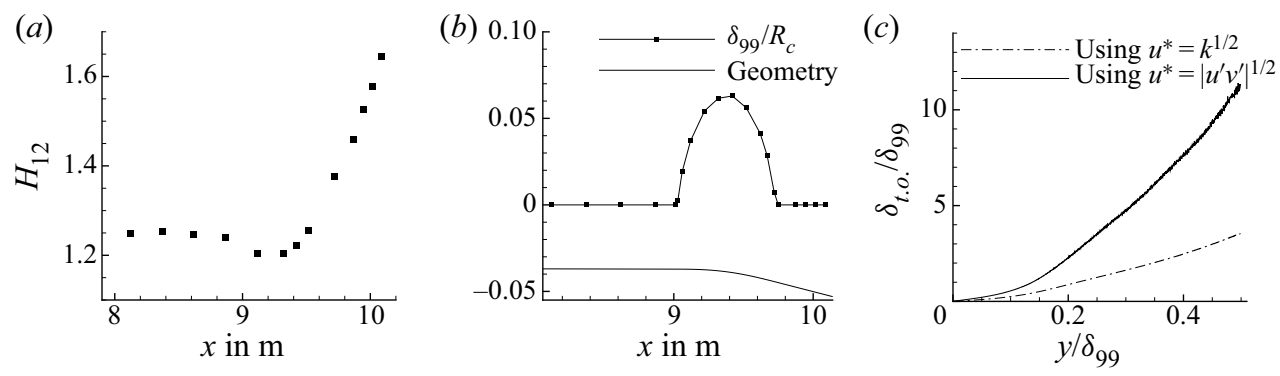

Figure 5. (a) Shape factor $H_{12}$ and (b) $\delta_{99} / R_{c}$ for $U_{\infty}=23 \mathrm{~m} \mathrm{~s}^{-1}$ (geometry not to scale), and (c) eddy turnover length $\delta_{t . o}$. for $U_{\infty}=36 \mathrm{~m} \mathrm{~s}^{-1}$.

reaches large positive values with a maximum near $x=9.62 \mathrm{~m}$ near the maximum of $\mathrm{d} c_{p} / \mathrm{d} s$. The shape factor $H_{12}$ is shown in figure 5(a). The local ratio of the boundary layer thickness $\delta_{99}$ to the radius of curvature $R_{c}$ is shown in figure $5(b)$. The values for $\delta_{99} / R_{c}$ are larger than the value of 0.01 which is associated with mild curvature in the literature. On the other hand, the value for $\delta^{*} / R_{c}$ is smaller than 0.005 , which is lower by one order 


\section{T. Knopp and others}

$\begin{array}{lcccccccccccc}\begin{array}{l}U_{\infty} \\ \mathrm{m} \mathrm{s}^{-1}\end{array} & \begin{array}{c}x \\ \mathrm{~m}\end{array} & \begin{array}{c}U_{e} \\ \mathrm{~m} \mathrm{~s}^{-1}\end{array} & R_{\theta} & R_{\tau} & \begin{array}{c}\delta_{99} \\ \mathrm{~mm}\end{array} & \begin{array}{c}\delta_{995} \\ \mathrm{~mm}\end{array} & \begin{array}{c}\delta^{*} \\ \mathrm{~mm}\end{array} & \begin{array}{c}\theta \\ \mathrm{mm}\end{array} & \begin{array}{c}H_{12} \\ \mathrm{~m} \mathrm{~s}_{\tau}\end{array} & \Delta p_{s}^{+} & \beta_{R C} \\ 23 & 8.120 & 28.13 & 24358 & 9304 & 147.6 & 161.0 & 16.77 & 13.42 & 1.250 & 0.977 & -0.00015 & -0.156 \\ 23 & 9.944 & 25.50 & 39822 & 6939 & 203.7 & 215.5 & 36.96 & 24.21 & 1.530 & 0.528 & 0.0185 & 27.06 \\ 36 & 8.120 & 43.29 & 35908 & 13214 & 142.2 & 157.2 & 16.06 & 12.88 & 1.247 & 1.433 & -0.00011 & -0.167 \\ 36 & 9.944 & 39.18 & 57363 & 9799 & 192.9 & 206.9 & 34.54 & 22.73 & 1.520 & 0.795 & 0.0114 & 26.37\end{array}$

Table 2. Characteristic boundary layer parameters for the 2D2C PIV measurements evaluated by the PIV single-pixel ensemble correlation method.

of magnitude than the criterion by Bradshaw (1970) for strong curvature. The boundary layer parameters are summarised in table 2 for the 2D2C PIV measurements evaluated by the single-pixel method.

The streamwise distance over which the pressure gradient changes may be compared to the boundary layer reference thickness, which is $\delta_{99, \text { ref }}=0.15 \mathrm{~m}$ at $x=8.12 \mathrm{~m}$. The curvature first causes an FPG from $x=8.85 \mathrm{~m}$ to $x=9.24 \mathrm{~m}$ over a streamwise length of $2.6 \delta_{99, \text { ref }}$. The change of $\beta_{Z S}$ from a significant FPG to a significant APG from $x=9.05 \mathrm{~m}$ to $x=9.62 \mathrm{~m}$ corresponds to $4 \delta 99$, ref. In the APG region on the inclined flat plate for $x>$ $9.75 \mathrm{~m}$, the curvature is absent and $\beta_{Z S}$ is slowly decreasing in the streamwise direction. The focus measurement position at $x=9.944 \mathrm{~m}$ in the APG region is located $1.3 \delta 99$, ref downstream of the end of curvature. For more insight, the local boundary layer thickness is related to the eddy turnover length and to the large-scale coherence.

The eddy turnover length $\delta_{\text {t.o. }}=U \tau_{\text {t.o. }}$ is the streamwise travelling distance of the local mean flow $U(y)$ corresponding to the eddy turnover time $\tau_{\text {t.o. }}$, see Sillero, Jimenez \& Moser (2013). Following this work, we assume that the flow relaxes to equilibrium within $2 \tau_{\text {t.o. }}$. We compute $\tau_{\text {t.o. }}$ using the relation $\tau_{\text {t.o. }}=\kappa y / u^{*}$, where two options were used for the turbulent velocity scale $u^{*}$, i.e. $u^{*}=\left|\overline{u^{\prime} v^{\prime}}\right|^{1 / 2}$ and $u^{*}=k^{1 / 2}$ based on the turbulent kinetic energy $k$. In the APG region at $x=9.944 \mathrm{~m}$, we observe $2 \delta_{\text {t.o. }}=0.5 \delta_{99}$ for $u^{*}=$ $k^{1 / 2}$ and $2 \delta_{t . o .}=1.1 \delta_{99}$ for $u^{*}=\left|\overline{u^{\prime} v^{\prime}}\right|^{1 / 2}$ at $y=0.1 \delta_{99}$, see figure $5(c)$. This is seen as an indication that the near-wall flow relaxes rapidly, but not instantaneously. In the outer part of the boundary layer, the turnover length becomes larger. At $y / \delta_{99}=0.5$ we observe $2 \delta_{\text {t.o. }}=22 \delta_{99}$ (based on $\left.\left|\overline{u^{\prime} v^{\prime}}\right|\right)$ and $2 \delta_{\text {t.o. }}=7 \delta_{99}$ (based on $\left.k\right)$.

An alternative streamwise length scale is the large-scale coherence in the flow. The largest values for the length scale from the two-point correlation map $L_{2}$ were found at the wall distance $y=0.2 \delta_{99}$ with $L_{2}=4 \delta_{99}$ at $x=8.34 \mathrm{~m}(\mathrm{ZPG}), L_{2}=4 \delta_{99}$ at $x=9.14 \mathrm{~m}$ (FPG) and $L_{2}=2 \delta_{99}$ at $x=9.94 \mathrm{~m}$ (APG), see Reuther (2019). Similar values were found for the length scale $L_{1}$ by Dennis \& Nickels (2011).

To summarise, the flow in the inner layer is expected to adjust rapidly, albeit not instantaneously, to the streamwise changing flow conditions as indicated from the eddy turnover length $\delta_{\text {t.o. }}$ and the large-scale coherence in the flow.

\subsection{Mean-velocity profile in the $Z P G$ region}

The mean-velocity profile at $x=8.12 \mathrm{~m}$ in the rear part of the flat plate before the flow enters the pressure gradient region is shown in figure 6(a). The experimental data for a canonical turbulent boundary layer flow at ZPG for a similar value of $R e_{\tau}$ and for a similar value of $\operatorname{Re}_{\theta}$ by Marusic et al. (2015) as for the present flow, see table 2, are included, 
(a)

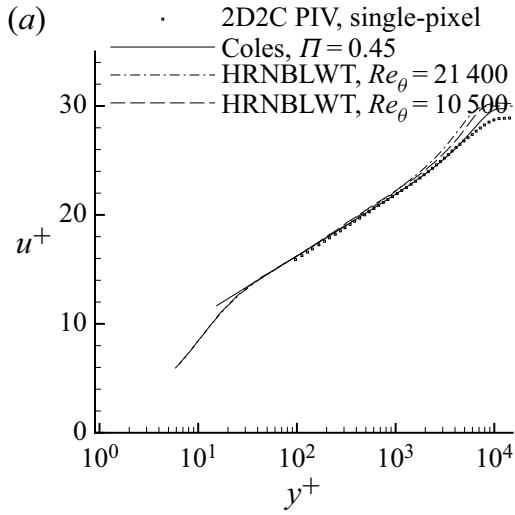

(b)

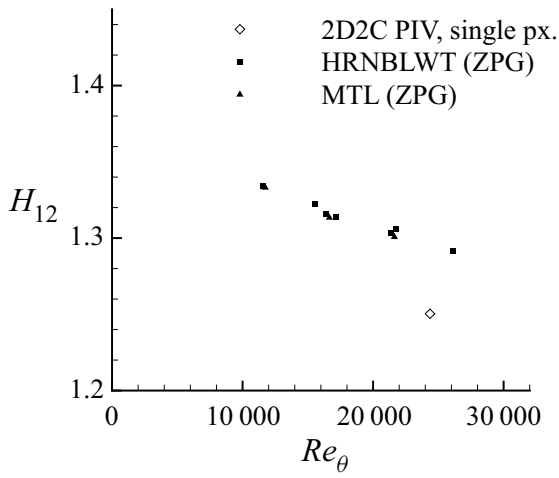

Figure 6. (a) Mean-velocity profile for $U_{\infty}=23 \mathrm{~m} \mathrm{~s}^{-1}$ at $x=8.12 \mathrm{~m}$ and reference data for a similar value of $R e_{\tau}$ and $R e_{\theta}$ in the wind tunnel HRNBLWT (high Reynolds number boundary layer wind tunnel at the University of Melbourne) by Marusic et al. (2015). (b) Shape factor $H_{12}$ and reference data in Bailey et al. (2013) and Marusic et al. (2015) measured in the wind tunnels HRNBLWT and MTL.

$\begin{array}{lccccccc}\begin{array}{l}U_{\infty} \\ \mathrm{m} \mathrm{s}^{-1}\end{array} & \text { PIV techn. } & \begin{array}{c}\text { Method } \\ \text { for } c_{f}\end{array} & 10^{4} \Delta p_{s}^{+} & \begin{array}{c}y_{\text {log,min }}^{+} \\ \text {interval }\end{array} & \begin{array}{c}y_{\text {log, } \max } / \delta_{995^{-}} \\ \text {interval }\end{array} & \begin{array}{c}u_{\tau} \\ \mathrm{m} \mathrm{s}^{-1}\end{array} & \kappa \\ 23 & \text { single px. } & \text { CCM } & -1.5 & {[100,800]} & {[0.1,0.15]} & 0.9681 & 0.3868 \\ 23 & \text { wind. corr. } & \text { CCM } & -1.5 & {[300,800]} & {[0.1,0.15]} & 0.9627 & 0.3987 \\ 36 & \text { single px. } & \text { CCM } & -1.1 & {[100,800]} & {[0.1,0.15]} & 1.4327 & 0.3916 \\ 36 & \text { wind. corr. } & \text { CCM } & -1.1 & {[500,800]} & {[0.1,0.15]} & 1.4270 & 0.4007\end{array}$

Table 3. Statistical evaluation of $u_{\tau}$ and $\kappa$ at $x=8.12 \mathrm{~m}$ at almost ZPG by variation of lower bound $y_{\text {log,min }}^{+}$ and upper bound $y_{\log , \max } / \delta_{995}$ assumed for the log-law region. px., pixel; wind. corr., window correlation.

together with the law of the wall by Coles with $\eta=y / \delta_{99}$ and $\Pi=0.45$

$$
u^{+}=\frac{1}{0.41} \log \left(y^{+}\right)+5.0+\frac{2 \Pi}{0.41}\left(\sin \left(\frac{\pi \eta}{2}\right)\right)^{2} .
$$

The wake of the present data is less pronounced than for the reference data. This is supposed to be a long-living history effect caused by the flow acceleration over the ramp. In the log-law region, the present data are close to the reference data. The small differences are supposed to be due to the small FPG and due to details in the method used to determine $u_{\tau}$. For the present data, the wall shear stress was determined from a Clauser chart method (CCM) using (i) $\kappa=0.41, B=5.0$, (ii) $\kappa=0.384, B=4.17$ and (iii) $\kappa=0.395, B=4.475$, and a variation of the log-law region $y_{\log , \min }^{+}<y^{+}<y_{\log , \max }^{+}$ for the statistical evaluation given in table 3. For the reference data, $u_{\tau}$ was determined using a composite velocity profile, see Marusic et al. (2015).

Then $\kappa$ and $B$ are determined by a least-squares fit of the data to the log law (1.1) using the value obtained for $u_{\tau}$. The results are given in table 3 . For a statistical evaluation, the log-law region was varied within the above intervals, leading to $\kappa=0.395 \pm 0.013$ for the mean value of $\kappa$ averaged over the four data sets in table 3. For the computation of $\delta^{*}$ and $\theta$, the mean-velocity profile by Chauhan, Monkewitz \& Nagib (2009) was used for $y^{+}$-values below the first reliable data point.

The shape factor $H_{12}$ evaluated at $x=8.12 \mathrm{~m}$ is shown in figure $6(b)$. For comparison, the data by Bailey et al. (2013) and Marusic et al. (2015), measured in the HRNBLWT and 


\section{T. Knopp and others}

in the minimum turbulence level wind tunnel (MTL) at the Royal Institute of Technology $(\mathrm{KTH})$, are included. For the present flow, $H_{12}$ is smaller than the reference data, consistent with the smaller wake factor, whereas $c_{f}=0.00244$ is larger than $c_{f}=0.00220$ for the reference data. We note that $c_{f}=2 u_{\tau}^{2} / U_{e}^{2}$ is normalised using the boundary layer edge velocity $U_{e}$ at $x=8.12 \mathrm{~m}$ for the present flow.

\subsection{Summary}

The experimental set-up provides the intended flow conditions. In the APG focus region, significant values of $\Delta p_{s}^{+}>0.01$ are reached and the flow is not close to separation. At the end of the ZPG region just upstream of the pressure gradient region, the mean velocity profile shows a well-defined log law in the inner layer and has a slightly smaller wake than a canonical flow. In the next section the detail measurements in the APG region and their analysis are presented.

\section{Results for the APG region}

The goal of this work is to find a description of the mean-velocity profile at APG in the inner layer and to answer questions (Q1)-(Q3). As classical inner scaling is used, summarised in appendix A.1, care is needed for the determination of the friction velocity $u_{\tau}$. The measurement position $x_{r e f}=9.944 \mathrm{~m}$ is in the middle of the inclined flat plate around $1.14 \delta_{99}$ downstream of the end of curvature. The $c_{f}$-distribution in figure $2(b)$ shows that the flow is far from separation at this station and further downstream.

\subsection{Determination of the wall shear stress}

The wall shear stress $\tau_{w}$ was determined using OFI. Additionally, $\tau_{w}$ was determined from the mean-velocity profiles. The definition of $\tau_{w}$ involves the mean-velocity gradient in wall-normal direction at the wall. The implication of this definition on the resolution requirements is still open for flows at a significant APG. Since the data points below $y^{+}=2$ were not considered reliable enough, different indirect methods were used based on a fit of the data to an assumed mean-velocity profile in a certain $y^{+}$-region. As a complementary method, $u_{\tau}$ was determined by a least-square fit of the total shear stress and the remaining terms of the integral momentum balance (A2), see Volino \& Schultz (2018).

\subsection{Mean-velocity profiles in the viscous sublayer}

The $\mu \mathrm{PTV}$ data for $U_{\infty}=23 \mathrm{~m} \mathrm{~s}^{-1}$ were considered to be reliable for $y^{+}>2$, and $u_{\tau}$ was determined by a least-squares fit to the relation $u^{+}=y^{+}$in the region $y^{+} \in$ $[2 \pm 0.1 ; 4.6 \pm 0.6]$. This region was found by visual comparison of the data with $u^{+}=y^{+}$and by inspection of $\partial U / \partial y$. The upper bound is a little lower than $y^{+}=5$ used by Nagano et al. (1991). We obtained $u_{\tau}=0.5217 \pm 0.0230 \mathrm{~m} \mathrm{~s}^{-1}$, compared to $u_{\tau}=0.5281 \pm 0.0106 \mathrm{~m} \mathrm{~s}^{-1}$ by OFI. The estimation of the relative uncertainty of $4.4 \%$ for $\mu \mathrm{PTV}$ is described in F.1, attempting to follow Bailey et al. (2014) for the different sources of uncertainties discussed in their work. The uncertainty due to a variation of the $y^{+}$-range used for the fit, and the uncertainty of the mean-velocity data per bin of the particle tracking method due to the number of sample events, were quantified using a Monte Carlo-based error analysis. The results for $u_{\tau}$ are summarised in table 4 . 
Method

for $u_{\tau}$

OFI

Fit $u^{+}=y^{+}$

Fit to Nickels (2004)

CCM $\begin{array}{cc}u_{\tau} & \epsilon_{u_{\tau}} \\ \text { in } \mathrm{m} \mathrm{s}^{-1} & \text { in } \%\end{array}$

$0.5281 \pm 0.0106$

$0.5217 \pm 0.0230$

$0.5270 \pm 0.0200$

$0.5037 \pm 0.0307$
2.0

4.4

3.8

6.1
$100 \Delta p_{s}^{+}$

$\epsilon_{\Delta p_{s}^{+}}$

in $\%$

10.6

18.3

16.3

23.9 $\epsilon_{\kappa}$

in $\%$

$0.3659 \pm 0.0252 \quad 6.9$

$0.3697 \pm 0.0233 \quad 6.3$

$0.3530 \pm 0.0304 \quad 8.6$

Table 4. Results for $u_{\tau}$ and $\kappa$ at the APG position for the $\mu \mathrm{PTV}$ data at $U_{\infty}=23 \mathrm{~m} \mathrm{~s}^{-1}$.

(a)

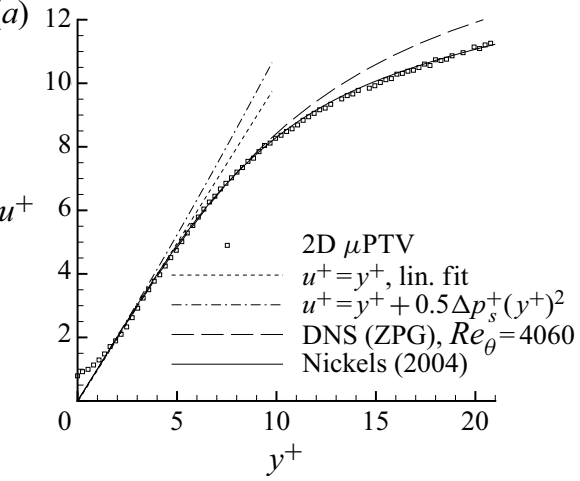

(b)

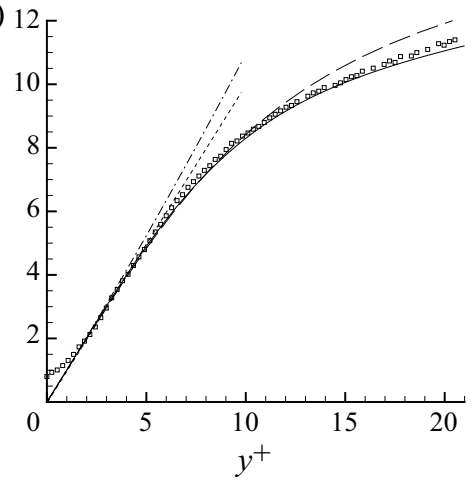

Figure 7. Two-dimensional $\mu \mathrm{PTV}$ data for $U_{\infty}=23 \mathrm{~m} \mathrm{~s}^{-1}$ at $x=9.944 \mathrm{~m}$. Mean-velocity profile in the viscous sublayer in inner units using $u_{\tau}(a)$ from OFI and $(b)$ from a linear fit to $u^{+}=y^{+}$.

The mean-velocity profiles are shown in figure 7 , where the inner scaling uses $u_{\tau}$ from OFI $(a)$ and from the fit $u^{+}=y^{+}(b)$. For $y^{+}<3$, the number of data points is not sufficient to show an advantage of the second-order Taylor-series expansion $u^{+}=$ $y^{+}+\frac{1}{2} \Delta p_{s}^{+}\left(y^{+}\right)^{2}$ for the present value of $\Delta p_{s}^{+}$. The mean-velocity profile (A4) by Nickels (2004) follows the $\mu$ PTV data very closely up to $y^{+}=20$. The DNS data for ZPG by Schlatter \& Örlü (2010) are close to the APG data near the wall, and the deviation increases for $y^{+}>10$, where the APG profile turns below the ZPG profile.

The profiles for $u^{+}$at $U_{\infty}=36 \mathrm{~m} \mathrm{~s}^{-1}$ are shown in figure 8 . The three-dimensional LPT data evaluation with the first reliable data point at $y^{+}=5$ was used, and $u_{\tau}$ was determined by a least-squares fit to the profile by Nickels (2004) for $y^{+}<21$, motivated by the findings for $U_{\infty}=23 \mathrm{~m} \mathrm{~s}^{-1}$. As a complementary method, $u_{\tau}$ was inferred from a least-square fit of the total shear stress and the remaining terms of the integral mean momentum balance (A2), see figure 16(b). This approach corresponds to the use of (3) in Volino \& Schultz (2018). The uncertainty for $u_{\tau}$ is estimated to be $5 \%$, see appendix E. As a cross-check, an empirical correction of the CCM for APG was used, motivated from the difference found between the CCM and the direct method for $u_{\tau}$ at $U_{\infty}=23 \mathrm{~m} \mathrm{~s}^{-1}$. The method is described in appendix G. The results for $u_{\tau}$ are summarised in table 5 .

\subsection{On the log law at $A P G$}

Regarding questions (Q1) and (Q2), a region where the mean velocity can be fitted by a $\log$ law was found by visual inspection of the plot $u^{+}$vs $\log \left(y^{+}\right)$for both cases 


\section{T. Knopp and others}
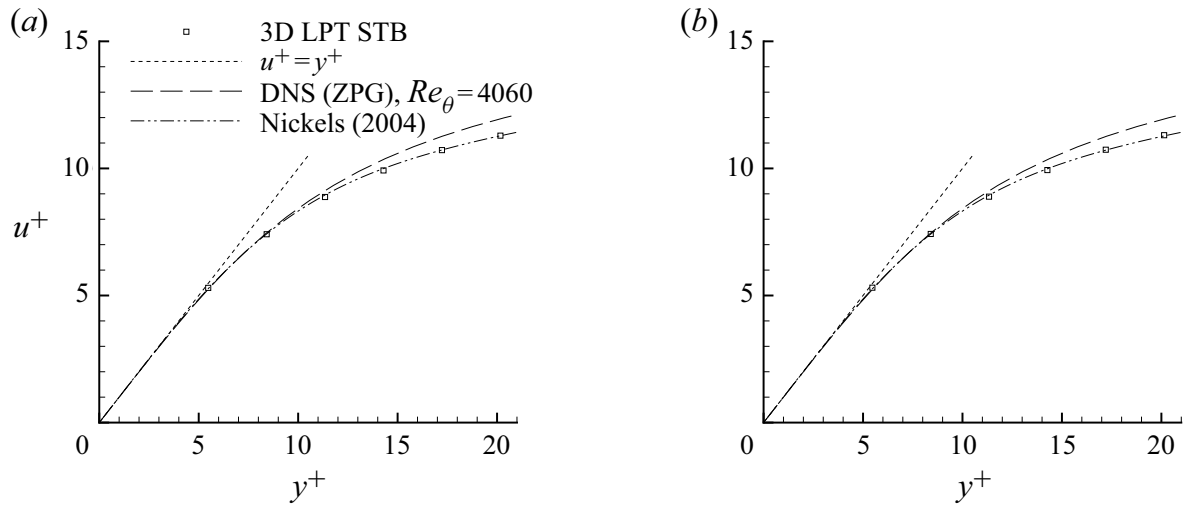

Figure 8. Three-dimensional LPT data for $U_{\infty}=36 \mathrm{~m} \mathrm{~s}^{-1}$ at $x=9.944 \mathrm{~m}$. Mean-velocity profile in the viscous sublayer in inner units using $u_{\tau}(a)$ from a least-squares fit of the total shear stress described in appendix $\mathrm{E}$ and $(b)$ from a least-squares fit to the mean-velocity profile by Nickels (2004).

$\begin{array}{lcccccc}\begin{array}{l}\text { Method } \\ \text { for } u_{\tau}\end{array} & \begin{array}{c}u_{\tau} \\ \text { in } \mathrm{m} \mathrm{s}^{-1}\end{array} & \begin{array}{c}\epsilon_{u_{\tau}} \\ \text { in } \%\end{array} & 100 \Delta p_{s}^{+} & \begin{array}{c}\epsilon_{\Delta p_{s}^{+}} \\ \text {in } \%\end{array} & \kappa & \begin{array}{c}\epsilon_{\kappa} \\ \text { in } \%\end{array} \\ \text { Fit to Nickels (2004) } & 0.7946 \pm 0.0318 & 4.0 & 1.144 \pm 0.194 & 17.0 & 0.3794 \pm 0.0197 & 5.2 \\ \text { Fit of total shear stress } & 0.7958 \pm 0.0398 & 5.0 & 1.139 \pm 0.231 & 20.3 & 0.3802 \pm 0.0259 & 6.2 \\ \text { CCM } & 0.7710 \pm 0.0431 & 5.6 & 1.253 \pm 0.279 & 22.3 & 0.3696 \pm 0.0251 & 6.8 \\ \text { CCM + corr. } & 0.7885 \pm 0.0441 & 5.6 & 1.171 \pm 0.261 & 22.3 & 0.3765 \pm 0.0256 & 6.8\end{array}$

Table 5. Results for $u_{\tau}$ and $\kappa$ at the APG position for the three-dimensional LPT data at $U_{\infty}=36 \mathrm{~m} \mathrm{~s}^{-1}$.

$U_{\infty}=23 \mathrm{~m} \mathrm{~s}^{-1}$ and $U_{\infty}=36 \mathrm{~m} \mathrm{~s}^{-1}$. Its extent is identified using the mean-velocity slope diagnostic function $(\mathrm{A} 8 a, b)$. For $U_{\infty}=36 \mathrm{~m} \mathrm{~s}^{-1}$, a thin plateau is found for $84<y^{+}<152$, see figure 16(a) in appendix D. For $U_{\infty}=23 \mathrm{~m} \mathrm{~s}^{-1}$ the log-law region is $86<y^{+}<135$.

The von Kármán constant $\kappa$ was determined using a log-linear regression. For $U_{\infty}=$ $23 \mathrm{~m} \mathrm{~s}^{-1}$, the lower bound $y_{\log , \min }^{+} \in[82 ; 92]$ and upper bound $y_{\log , \max }^{+} \in[126 ; 138]$ were varied for statistical evaluation. The results are given in table 4 . The value $\kappa=0.370 \pm$ 0.017 at $\Delta p_{s}^{+}=0.0183$ was obtained for $u_{\tau}$ by OFI. Beyond the bounds of the $y^{+}$-range, the values for $\kappa$ change significantly due to the systematic deviation of the mean-velocity profile from a log-linear behaviour, in particular for $y^{+}$much larger than 140. The log-law fit is shown in figure $9(a)$. The uncertainty estimation is described in appendix F.2 and summarised in table 12. It was guided by the work of Bailey et al. (2014).

For $U_{\infty}=36 \mathrm{~m} \mathrm{~s}^{-1}, y_{\log , \min }^{+} \in[82 ; 90]$ and $y_{\log , \max }^{+} \in[142 ; 150]$ were used. The value of $\kappa=0.379 \pm 0.020$ at $\Delta p_{s}^{+}=0.0114$ was obtained for $u_{\tau}$ determined by the least-squares fit to the profile by Nickels. The detailed results are given in table 5 . The mean-velocity profile and the log-law fit are shown in figure $9(b)$. A detailed discussion of the uncertainties and their quantification is given in appendix F.2 and in table 13 .

An important finding is that the outer limit of the log law is at a much smaller $y^{+}$-value than for ZPG flows, i.e. at $0.019 \delta_{99}^{+}$for $U_{\infty}=23 \mathrm{~m} \mathrm{~s}^{-1}$ and at $0.015 \delta_{99}^{+}$for 

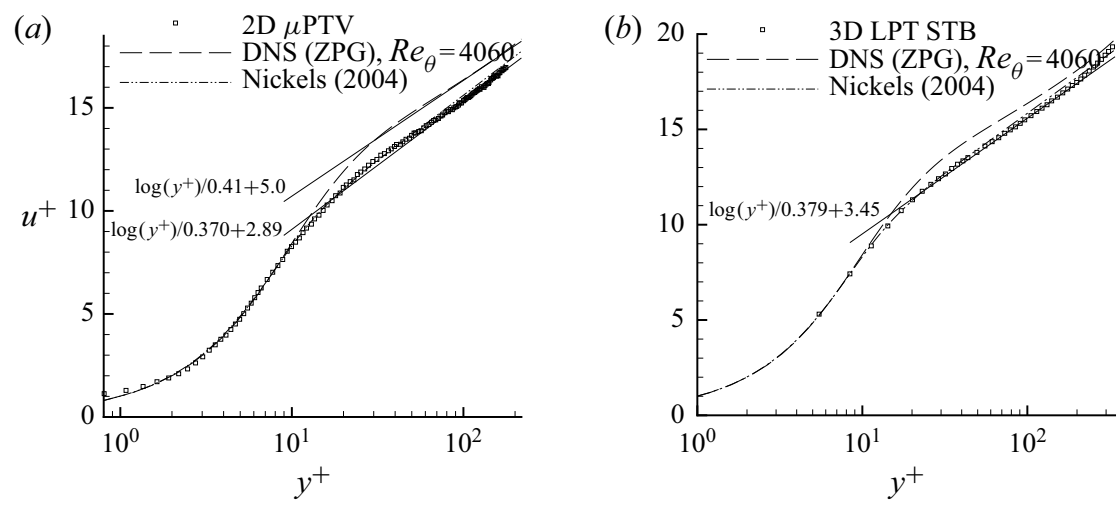

Figure 9. Mean-velocity profile and least-squares fit in the log-law region at $x=9.944 \mathrm{~m}(a)$ for $U_{\infty}=$ $23 \mathrm{~m} \mathrm{~s}^{-1}$ using $u_{\tau}$ from OFI and (b) for $U_{\infty}=36 \mathrm{~m} \mathrm{~s}^{-1}$ using $u_{\tau}$ from a least-squares fit to the profile by Nickels (2004) for $y^{+}<20$.

$U_{\infty}=36 \mathrm{~m} \mathrm{~s}^{-1}$. It is even smaller than the widely believed start of the log-law region in ZPG flows, cf. Wei et al. (2005) and Marusic et al. (2013). This indicates that, at a given significant value of, say, $\Delta p_{s}^{+}>0.01$, the extent of the log law cannot be simply increased by increasing $R e$. This is different to the traditional view for ZPG, where the log law is associated with an overlap region, whose extent in terms of $y^{+}$can be increased by increasing $R e$. At APG, a half-power law is found instead in a large part of the overlap region, and its extent can be increased by increasing $R e$, see $\S 4.6$.

\subsection{Clauser chart method}

For the study of the CCM at APG, the same statistical evaluation as in $\S 4.3$ was used. For $U_{\infty}=23 \mathrm{~m} \mathrm{~s}^{-1}, u_{\tau}=0.5037 \mathrm{~m} \mathrm{~s}^{-1}$ is obtained. The sensitivity of $u_{\tau}$ to the values for $\kappa$ and $B$ calibrated for ZPG is smaller than $0.3 \%$, given that the upper and lower bounds for the log-law region are fixed. The uncertainties are summarised in table 14 in appendix F.3. For $U_{\infty}=36 \mathrm{~m} \mathrm{~s}^{-1}, u_{\tau}=0.7710 \mathrm{~m} \mathrm{~s}^{-1}$ is obtained, and the uncertainties are given in table 15 . The values for $u_{\tau}$ by the Clauser chart are systematically lower than from OFI and from the viscous sublayer fit, in agreement with Monty et al. (2011).

\subsection{Von Kármán constant and log-law intercept}

The values for $\kappa$ and $B$ are correlated with the pressure gradient parameter. In figure 10(a) $\kappa$ is plotted vs $\Delta p_{s}^{+}$, as proposed by Nickels (2004), see correlation (A7). The error bars for both quantities $\Delta p_{s}^{+}$and $\kappa$ are also included. The details of the uncertainty analysis are described in appendix F.2. The uncertainty of $\Delta p_{s}^{+}$uses an assumed uncertainty of $3.5 \%$ for $(1 / \rho)\left(\mathrm{d} P_{\mathrm{w}} / \mathrm{d} s\right)$ and an uncertainty of $1 \%$ for $v$. The symbols indicate the method used to determine $u_{\tau}$. The values for $\kappa$ are found to be smaller for $U_{\infty}=23 \mathrm{~m} \mathrm{~s}^{-1}$ than for $U_{\infty}=36 \mathrm{~m} \mathrm{~s}^{-1}$. This is consistent with the proposal by Nickels in the sense that $\kappa$ is decreasing with increasing values of $\Delta p_{s}^{+}$. For the reference value at ZPG, $\kappa=0.40 \pm$ 0.02 is assumed. Thus a reduction of $\kappa$ at APG is found compared to $\kappa=0.40$, but this reduction is within the estimated uncertainty bounds.

Moreover, a significant role of the method to determine $u_{\tau}$ is observed. The standard CCM yields lower values for $u_{\tau}$, and hence larger values for $\Delta p_{s}^{+}$, and smaller values for 


\section{T. Knopp and others}
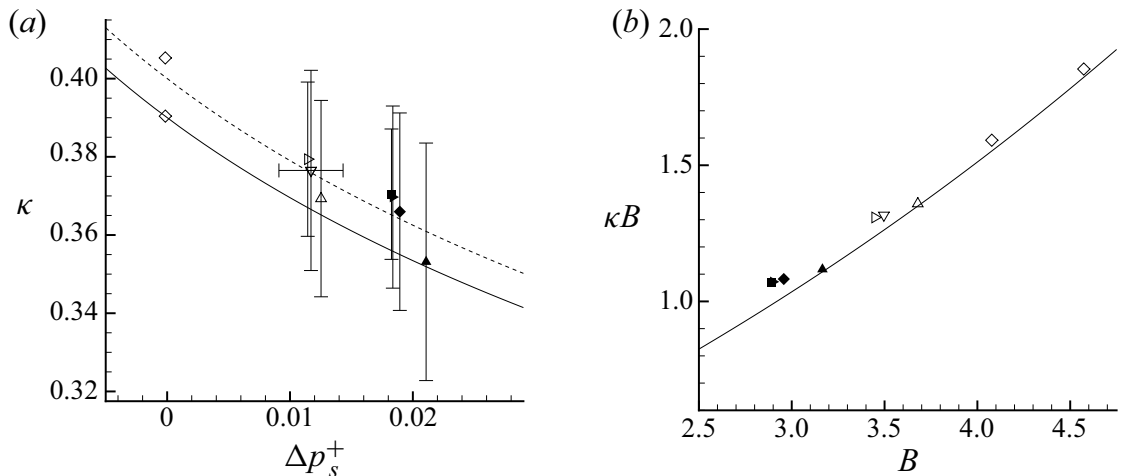

Figure 10. (a) Value of $\kappa$ vs $\Delta p_{s}^{+}$. The symbols show the method for $u_{\tau}$. Two-dimensional $\mu$ PTV data for $U_{\infty}=23 \mathrm{~m} \mathrm{~s}^{-1}: \mathbf{\square}$, OFI; $\downarrow$, fit to $u^{+}=y^{+}$; $\boldsymbol{\nabla}$, fit to profile by Nickels (2004); $\mathbf{\Delta}$, Clauser chart. Three-dimensional LPT data for $U_{\infty}=36 \mathrm{~m} \mathrm{~s}^{-1}: \triangle$, Clauser chart; $\triangleright$, fit to profile by Nickels (2004); $\nabla$, Clauser chart with empirical correction. The 2D2C PIV data using OFI for $u_{\tau}: \diamond, x=8.12 \mathrm{~m}$ at almost ZPG; solid line, correlation by Nickels (2004) for $\kappa_{0}=0.39$; dashed line, correlation by Nickels (2004) using $\kappa_{0}=0.40$. (b) Plot of $\kappa B$ and vs $B$. Same symbols as in (a); solid line, correlation by Monkewitz et al. (2008).

$\kappa$ are obtained. This gives a warning that a possible change of $\kappa$ may appear enlarged, if a standard CCM is used to determine $u_{\tau}$.

Regarding the intercept $B$, the values for $\kappa B$ are plotted against $B$ in figure $10(b)$ and follow closely the correlation proposed by Monkewitz et al. (2008).

\subsection{On the half-power-law region}

Then the hypothesis of a half-power-law region (or sqrt-law region) above the log law is studied, see question (Q3) in the introduction. The mean velocity slope diagnostic function $(\mathrm{A} 8 a, b)$ is used to identify the region where the mean-velocity profile can be described by the sqrt law (1.3) following the method for ZPG turbulent boundary layers by Österlund et al. (2000) and Monkewitz, Chauhan \& Nagib (2007). For $U_{\infty}=23 \mathrm{~m} \mathrm{~s}^{-1}$, the mean-velocity profile and the fit to the sqrt law are shown in figure $11(a)$ for the 2D2C PIV data evaluated by the single-pixel ensemble correlation method and $u_{\tau}$ from OFI. The least-squares fit of (1.3) to the data is computed in the region where the slope diagnostic function $(\mathrm{A} 8 a, b)$ gives a small plateau, see figure $11(b)$. The single-pixel data show an approximative plateau in the region $500<y^{+}<860$, whereas the window correlation data are smoother and show a slightly smaller plateau for $520<y^{+}<840$. The outer edge of the approximative plateau $y^{+} \approx 840$ corresponds to $0.122 \delta_{99}^{+}$.

For $U_{\infty}=36 \mathrm{~m} \mathrm{~s}^{-1}$ the mean-velocity profile and the fit to the sqrt law are shown in figure 12(a) for the three-dimensional LPT data. A region of an approximative plateau for the slope diagnostic function (A8a,b) can be observed for $y^{+}>750$ which extends up to approximately $y^{+} \approx 1260$, see figure $12(b)$. The outer edge $y^{+} \approx 1260$ corresponds to $0.129 \delta_{99}^{+}$. The outer limit of the plateau in terms of $y^{+}$is increasing with increasing $U_{\infty}$, i.e. with increasing $R e$. This is similar to the behaviour of the outer edge of the log law in turbulent boundary layers at ZPG. We note that the mean-velocity profile cannot be fitted by the sqrt law (1.3) in the entire inner region, say, $y^{+} \in\left[150,0.13 \delta_{99}^{+}\right]$.

The outer limit of the plateau is smaller than $y / \delta=0.15$ found for ZPG by Marusic et al. (2013), where the boundary layer thickness $\delta$ was determined from a fit of the 
(a)

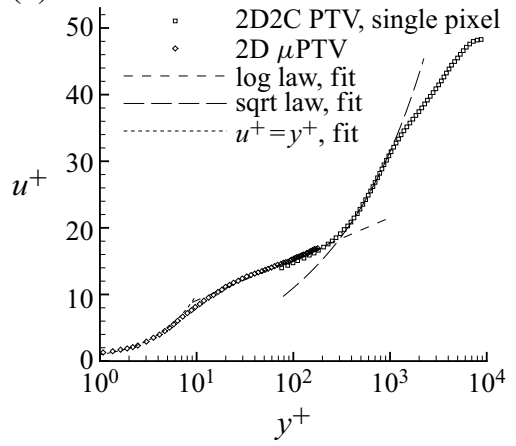

(b)

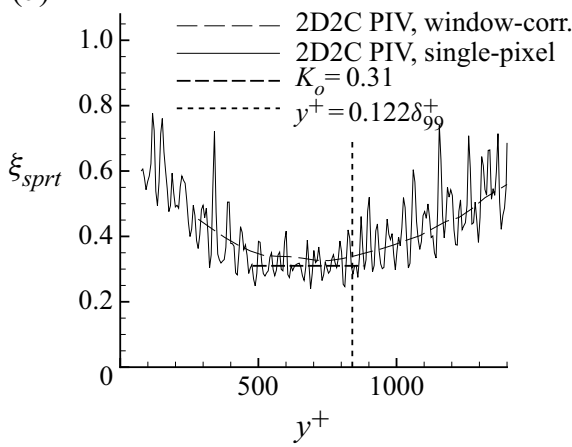

Figure 11. The 2D2C PIV results at APG for $U_{\infty}=23 \mathrm{~m} \mathrm{~s}^{-1}$ at $x=9.944 \mathrm{~m}$. (a) Log-law and sqrt-law regions. (b) Mean-velocity slope diagnostic function of the sqrt law.
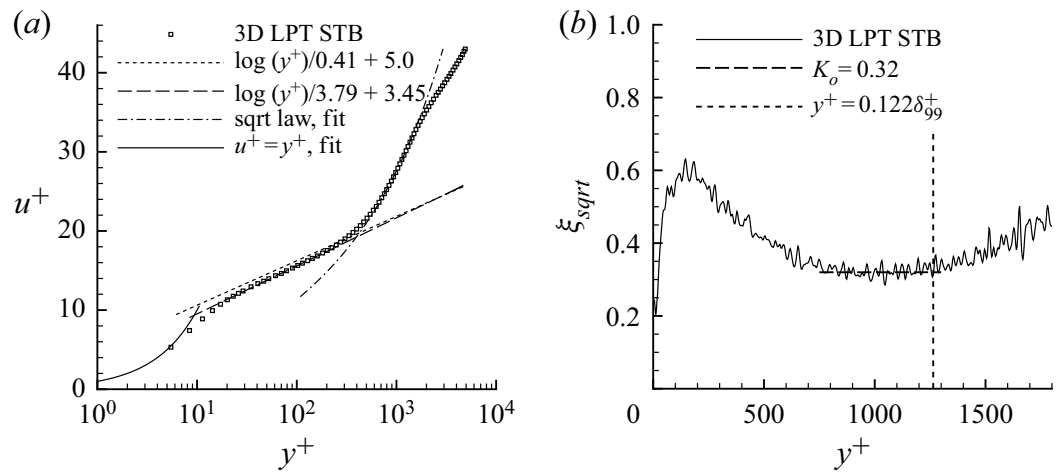

Figure 12. Three-dimensional LPT STB results for $U_{\infty}=36 \mathrm{~m} \mathrm{~s}^{-1}$ at $x=9.944 \mathrm{~m}$. (a) Log-law and sqrt-law regions. (b) Mean-velocity slope diagnostic function of the sqrt law.

composite law of the wall/law of the wake. The value $0.15 \delta$ given in Marusic et al. (2013) corresponds to $0.195 \delta_{99}$ if assuming that $\delta=1.3 \delta_{99}$. An estimate of historical effects using $2 \delta_{\text {t.o. }}$ based on $u^{*}=k^{1 / 2}$ in figure $5(c)$ gives $2 \delta_{\text {t.o. }}=0.8 \delta_{99}$ at $y=0.13 \delta_{99}$, $2 \delta_{\text {t.o. }}=1.0 \delta_{99}$ at $y=0.15 \delta_{99}$ and $2 \delta_{\text {t.o. }}=1.7 \delta_{99}$ at $y=0.2 \delta_{99}$. Therefore, the outer limit of the plateau of $\xi_{\text {sqrt }}$ and the deviation of the mean-velocity profile from the sqrt-law fit above $y^{+} \geqslant 0.15 \delta_{99}$ could be affected by historical effects, leading to a smaller outer limit of the sqrt law than for the log law at ZPG, see $\S 5$. The inner limit of the plateau is larger than the value $y^{+}=350$ inferred from the relation $y^{+}=3 R e_{\tau}^{1 / 2}$ for ZPG by Marusic et al. (2013), assuming again $R e_{\tau}=\delta^{+} \approx 1.3 \delta_{99}^{+}$.

Regarding the significant variation in scatter for figures $11(b)$ and $12(b)$, we note that the gradients for the 2D2C PIV data in figure 11(b) are computed using a central difference scheme for the unfiltered data. For the three-dimensional LPT data in figure $12(b)$, a two-step method was used. In the first step a smoothing of the data was applied, using a Gaussian filter with a kernel of 7 points, i.e. using the data points $i-3, \ldots, i+3$ for data point $i$. To compute the gradient at $y=y_{i}$, a linear function was fitted to the data points $y_{i-3}, \ldots, y_{i}, \ldots, y_{i+3}$ using a least-square fit. Note that $y_{i+3}-y_{i}=8.7 \delta_{v}$ can be seen as the half filter width for smoothing, see also appendix D.

The mean-velocity profile above the square-root-law region looks different from the wake of a turbulent boundary layer at APG on a flat plate, see figures $11(a)$ and $12(a)$. 


\section{T. Knopp and others}

This originates from the upstream region of convex surface curvature and streamwise changing pressure gradient and will be described in $\S 5$.

The value for $K_{o}$ was determined by a least-square fit to (1.3), yielding $K_{o}=0.310$ and $B_{o}=-6.39$ for the single-pixel data at $U_{\infty}=23 \mathrm{~m} \mathrm{~s}^{-1}$ and $u_{\tau}$ from OFI, and $K_{o}=0.320$ and $B_{o}=-4.66$ for the three-dimensional LPT STB data at $U_{\infty}=36 \mathrm{~m} \mathrm{~s}^{-1}$, where $u_{\tau}$ from the least-squares fit to the profile by Nickels was used. For the comparison of $K_{o}$ with values reported in the literature, note that some authors use the form for the half-power law for zero-skin-friction flow by Stratford (1959)

$$
U(y)=\frac{2}{K}\left(\frac{1}{\rho} \frac{\mathrm{d} P}{\mathrm{~d} s}\right)^{1 / 2} y^{1 / 2}
$$

instead of (1.3). Stratford used a value of $K=0.66 \kappa=0.27$ for his zero-skin-friction flow. For the same data, Townsend (1960) found $K=0.5 \pm 0.05$, whereas Mellor (1966) found that, at most, $K=0.44$ by excluding certain near-wall points. From the DNS by Spalart \& Leonard (1986), $K=0.6$ was found. For flows with a non-zero skin friction, some representative values are $0.48 \pm 0.03$ by Townsend (1961), 0.48 by Perry (1966), for large values of $\left(\delta / \tau_{w}\right)(\mathrm{d} P / \mathrm{d} s), 0.45$ by Kader $\&$ Yaglom (1978) and 0.57 by Afzal (2008). The values depend on the form of the half-power law, i.e. (1.3) or (4.1), and on the region considered for the least-squares fit. For example, if the half-power-law fit is applied above the log-law region, as proposed by Perry (1966), then smaller values are obtained than if the fit is applied to all data in the inner layer above the buffer layer. The latter method was used e.g. by Afzal (2008). The values obtained for $K_{O}$ for the present flow are smaller than other values reported in the literature, despite these details.

As a final remark, Perry (1966) proposed an extension of his three-layer model, suggesting that the first and second streamwise derivatives of the pressure gradient might need to be included to account for effects of a streamwise decreasing APG. He proposed a region IV above the half-power-law region, where the mean-velocity gradients are proportional to $y^{0}$ (compared to $y^{-1}$ in the log-law region and $y^{-1 / 2}$ in the half-power-law region) and are governed by $\mathrm{d}^{2} P / \mathrm{d} s^{2}$. Such a region was also proposed by Durbin \& Belcher (1992) for reasons of matching with the outer part of the boundary layer and called the middle region. For the present data, a region where $U \sim y$ can be observed from $y^{+}=3500$ to $y^{+}=5000$, corresponding to $0.35<y / \delta_{99}<0.51$.

\subsection{Summary}

The log law in the mean-velocity profile was found to be a robust feature at APG, but its region is thinner than in zero pressure gradient flows (Q1). The von Kármán constant $\kappa$ was found to be reduced, but within the uncertainty of the measurement (Q2). A square-root law was observed to emerge above the log law in a large part of the region the log law occupies at ZPG (Q3).

\section{Streamwise evolution of the mean velocity}

As the focus is on the mean flow scaling and given that the inner and outer regions are connected by an overlap region, history effects for the inner and outer layer need to be discussed. Perry et al. (1966) divided the boundary layer into a 'wall region' and a 'historical region'. In the wall region, only the local flow quantities $\mathrm{d} P / \mathrm{d} s, \tau_{w} / \rho$, and $y$ govern the mean-velocity profile, and higher derivatives of $\mathrm{d} P / \mathrm{d} s$ and $\tau_{w} / \rho$ may be involved above a certain wall distance. In the historical region, the mean-velocity profile 


$\begin{array}{lcccccccccccc}x \text { in } \mathrm{m} & 8.12 & 8.62 & 8.87 & 9.12 & 9.32 & 9.42 & 9.52 & 9.72 & 9.87 & 9.944 & 10.02 & 10.09 \\ x_{\text {curv }}^{*} & -5.80 & -2.47 & -0.80 & 0.86 & 2.20 & 2.86 & 3.53 & 4.86 & - & - & - & - \\ x_{\text {relax }}^{*} & - & - & - & - & - & - & - & -0.20 & 0.70 & 1.14 & 1.59 & 2.00 \\ 100 \Delta p_{s}^{+} & -0.015 & -0.046 & -0.095 & -0.17 & 0.037 & 0.20 & 0.49 & 1.34 & 1.57 & 1.85 & 2.26 & 2.75 \\ \beta_{R C} & -0.16 & -0.52 & -1.11 & -1.73 & 0.30 & 1.63 & 4.50 & 14.9 & 18.4 & 27.1 & 35.3 & 47.0\end{array}$

Table 6. Positions for the mean-flow profiles from the 2D2C PIV overview measurement. The values are for the case $U_{\infty}=23 \mathrm{~m} \mathrm{~s}^{-1}$ using the values for $u_{\tau}$ obtained by OFI.
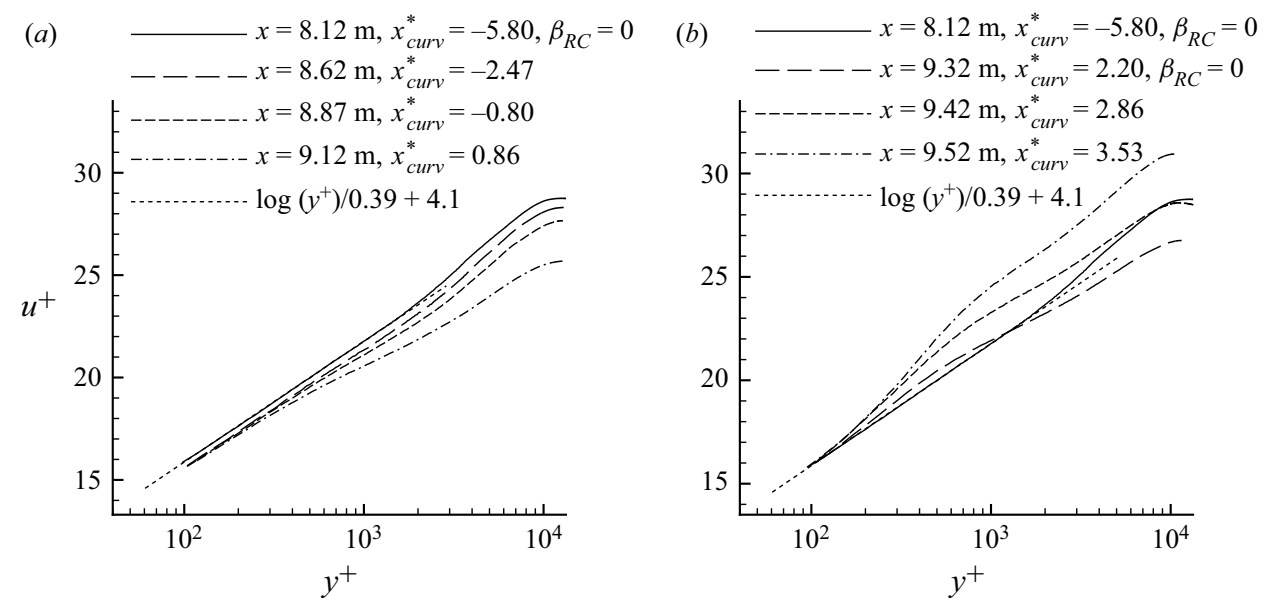

Figure 13. Mean-velocity profiles for $U_{\infty}=23 \mathrm{~m} \mathrm{~s}^{-1}$ at almost ZPG and in the region of curvature with change from favourable to APG.

is influenced by 'upstream events'. To better understand the mean-velocity profile at the APG focus position $x=9.944 \mathrm{~m}$, the local effects and the historical effects of streamwise changing pressure gradient, convex curvature and relaxation from curvature are discussed as the flow evolves downstream. The history effects in the outer region are quantified using the shape factor $H_{12}$ due to the issues in the definition of the wake parameter, see Chauhan et al. (2009) for ZPG flows. Table 6 gives the streamwise positions studied. The distance $x_{\text {curv }}^{*}=\left(x-x_{b}\right) / \delta 99$, ref from the beginning of curvature at $x_{b}=8.990 \mathrm{~m}$ is defined as a multiple of $\delta_{99, \text { ref }}$, and $x_{\text {relax }}^{*}=\left(x-x_{e}\right) / \delta_{99, \text { apg }}$ defines the distance of flow relaxation downstream of the end of curvature, measured from the end of curvature at $x_{e}=9.750 \mathrm{~m}$, as a multiple of $\delta_{99, \text { apg }}=0.17 \mathrm{~m}$ at $x=9.72 \mathrm{~m}$.

The change from ZPG to FPG causes a reduction of $H_{12}$ and of the wake factor $\Pi$, which is known for FPG, see, e.g. Jones, Marusic \& Perry (2001). Moreover, a downward shift of the entire $u^{+}$-profile by $\Delta u^{+}=-0.4$ is observed, see figure 13(a). This shift is not observed if $u_{\tau}$ from the CCM is used, giving $2 \%$ to $3 \%$ larger values for $u_{\tau}$, close to the uncertainty of $2 \%$ of the OFI method. As the FPG becomes strongest near $x=9.12 \mathrm{~m}$ and curvature effects increase, the extent of the log-law region is observed to be reduced to $y^{+}<450$ (or $y / \delta_{99}<0.047$ ), and above, the velocity profile, plotted against $\log \left(y^{+}\right)$, turns downwards below the $\log$ law. Regarding the reduction of $\Pi$, the acceleration parameter $K=-v / U_{e}^{2} \mathrm{~d} U_{e} / \mathrm{d} s$ quantifying the strength of the FPG is 


\section{T. Knopp and others}

(a)

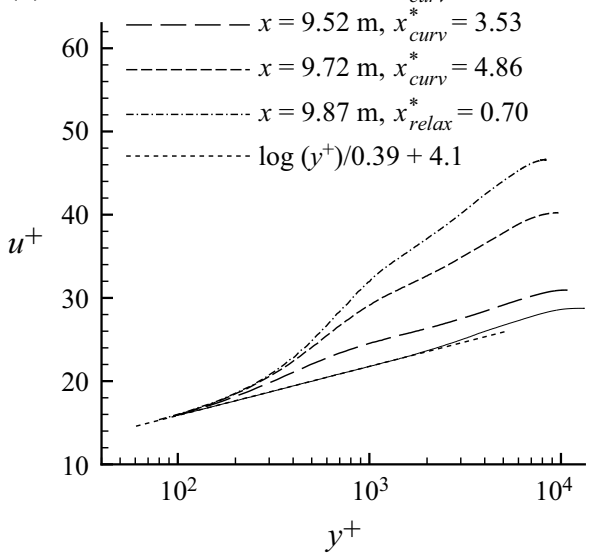

(b)

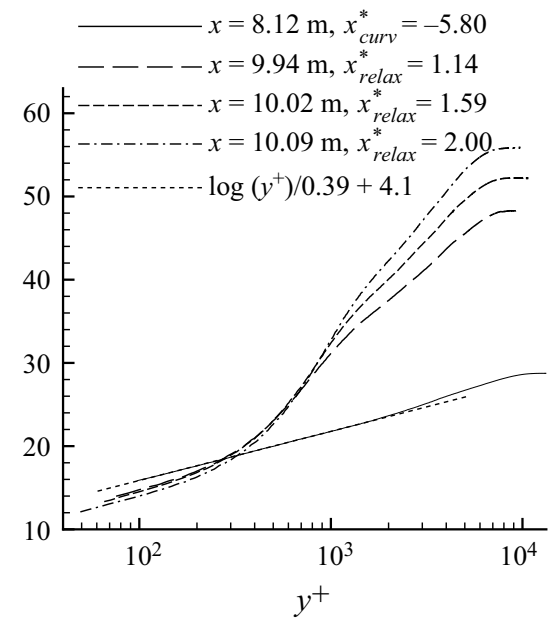

Figure 14. Mean-velocity profiles for $U_{\infty}=23 \mathrm{~m} \mathrm{~s}^{-1}$ in the APG region.

$K=1.0 \times 10^{-7}$. Hence, $K$ and $\Delta p_{s}^{+}$are smaller than for the smallest FPG case $K=$ $2.7 \times 10^{-7}$ and $\Delta p_{s}^{+}=-3.3 \times 10^{-3}$ in Jones et al. (2001). Then the pressure gradient rapidly changes from increasing to decreasing FPG. History effects in the outer layer are found at $x=9.32 \mathrm{~m}$, where $\beta_{R C}$ is (almost) zero. Here $H_{12}$ remains at the low value of $H_{12}=1.20$ at $x=9.12 \mathrm{~m}$ and is significantly smaller than $H_{12}=1.25$ in the upstream ZPG region.

The APG region on the curved wall $(x \geqslant 9.32 \mathrm{~m})$ is shown in figure $13(b)$. In the inner layer, the value of $u^{+}$at $y^{+}=100$, denoted by $u^{+}(100)$, remains almost unaltered. For $y^{+}>200$ (or $y / \delta_{99}>0.024$ ), the profiles begin to turn upwards above the log law. This is mainly attributed to the APG, which reaches $\Delta p_{s}^{+}=0.005$ at $x=9.52 \mathrm{~m}$. Note that a reduction of the log-law region and an upward turn above the log law was also observed in convex curvature flows at ZPG by Gillis et al. (1980), and by Kim \& Rhode (2000). However, the upward turn, quantified by $\Delta u^{+}\left(y^{+}\right)$above the log law, shown therein is smaller than for the present flow. Regarding the outer layer, $H_{12}$ is rising downstream of $x>9.32 \mathrm{~m}$ due to the APG, albeit convex curvature was also found to cause a small increase of $H_{12}$, see Patel \& Sotiropoulos (1997). Another historical effect is found at $x=9.52 \mathrm{~m}$, where $H_{12}$ reaches again the value from the ZPG region, albeit at $\beta_{R C}=4.5$ and with a different form of the mean-velocity profile.

Downstream of the end of curvature at $x=9.750 \mathrm{~m}$, the flow is subjected to an APG and relaxation from curvature. The mean-velocity profiles are shown in figure 14(a). Regarding the inner layer, the value for $u^{+}(100)$ appears still to be almost unchanged. The upward turn above the log law is increasing in downstream direction, consistent with the increase of the slope of the square-root law with increasing $\Delta p_{s}^{+}$. For $x \geqslant 9.944 \mathrm{~m}, u^{+}(100)$ is found to be decreasing, and in the log-law region the profiles appear to be shifted below the log law at ZPG, see figure 14(b). The log-law region appears to recover as the flow relaxes from curvature. The recovery for the ZPG case is described in Gillis et al. (1980) and Alving et al. (1990). In the square-root-law region, the slope of $u^{+}$is continuously increasing, consistent with the increasing $\Delta p_{s}^{+}$values. Regarding the outer layer, $\Pi$ and $H_{12}$ are further increasing due to the APG, but remain substantially smaller than for the corresponding equilibrium flow. 
(a)

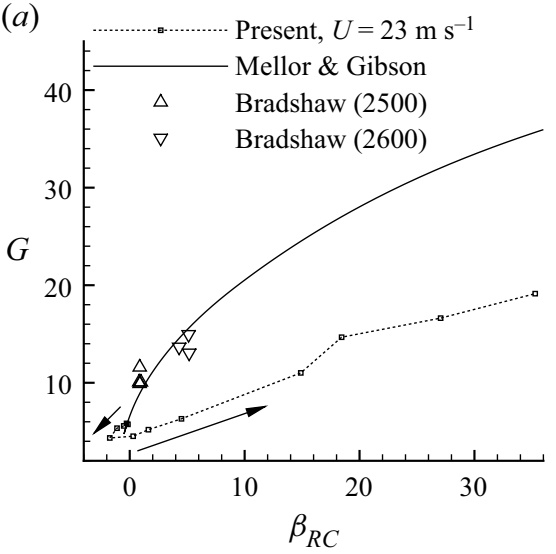

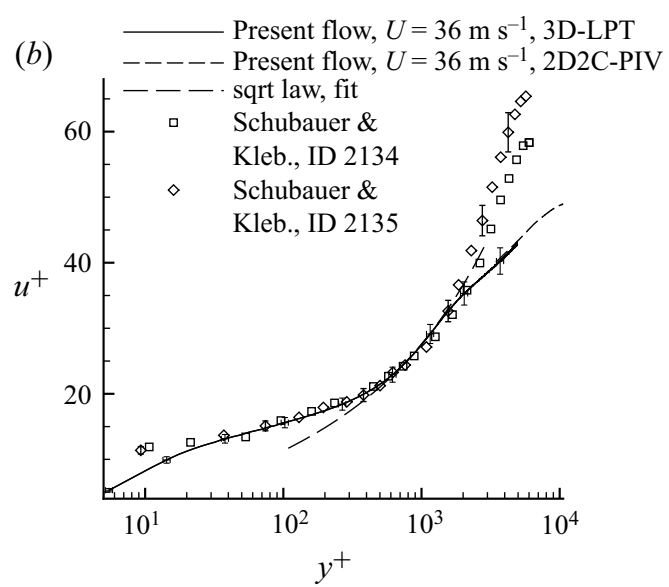

Figure 15. (a) Defect shape factor $G$ for $U_{\infty}=23 \mathrm{~m} \mathrm{~s}^{-1}$ and theory for equilibrium flows by Mellor \& Gibson (1966) with the data by Bradshaw in Coles \& Hirst (1969). (b) Mean velocity for $U_{\infty}=36 \mathrm{~m} \mathrm{~s}^{-1}$ at $x=$ $9.944 \mathrm{~m}$ and data by Schubauer \& Klebanoff in Coles \& Hirst (1969) at similar local flow conditions $\Delta p_{s}^{+}$and $\Delta u_{\tau, s}^{+}$, see $(\mathrm{E} 2 a-c)$.

$\begin{array}{lcccccc}\text { Author } & \text { ID } & R e_{\theta} & \Delta p_{s}^{+} & \Delta u_{\tau, s}^{+} \times 10^{5} & \beta_{R C} & H_{12} \\ \text { Present, } U_{\infty}=36 \mathrm{~m} \mathrm{~s}^{-1} & 9.944 \mathrm{~m} & 57363 & 0.0114 & -1.974 & 26.37 & 1.520 \\ \text { Schubauer \& Klebanoff } & 2134 & 53838 & 0.0117 & -2.55 & 20.286 & 1.857 \\ \text { Schubauer \& Klebanoff } & 2135 & 58117 & 0.0147 & -4.57 & 27.291 & 1.983\end{array}$

Table 7. Summary of local flow parameters for APG flows shown in figure $15(b)$.

The cumulative history effect of the present flow is studied in terms of the defect shape factor $G$, where $H_{12}=(1-\gamma G)^{-1}$ with $\gamma=u_{\tau} / U_{e}$ to account for Re-effects, and compared with the theory for equilibrium turbulent boundary layer flows by Mellor \& Gibson (1966). Together with the streamwise distribution of $\beta_{\mathrm{RC}}$ given in figure $4(b)$, figure 15(a) shows the delayed response of $G$ for the present flow compared to the corresponding equilibrium flow at the same value of $\beta_{R C}$.

Between the outer edge of the square-root law and the wake, an $S$-shaped bending in the $u^{+}$-profile is observed, if plotted against $\log \left(y^{+}\right)$, appearing at $x=9.42 \mathrm{~m}$ and persisting downstream. From the flow history, the $S$-shaped bending appears as a combined effect of the upward turn above the log law due to the APG, starting to grow for $x \geqslant 9.42 \mathrm{~m}$, and the downward turn below the log law originating in the upstream FPG region. This could be a non-equilibrium effect due to the rapid change in $u_{\tau}$ and in $\mathrm{d} P / \mathrm{d} s$ over a short streamwise distance, see Spalart (2010).

Finally, the role of local and historical effects on the mean velocity in the inner layer are discussed for the high-Re case, given that the inner and outer layer are connected by an overlap region. For the local effects $\Delta p_{s}^{+}$and the wall shear stress gradient parameter $\Delta u_{\tau, s}^{+}$are considered, motivated by the model for the total shear stress $(\mathrm{E} 2 a-c)$, where $\Delta u_{\tau, s}^{+}$describes the local flow acceleration. Figure 15(b) compares the present data with two profiles by Schubauer \& Klebanoff (denoted by 2134 and 2135 in Coles \& Hirst 1969) at almost the same values of $\Delta p_{s}^{+}, \Delta u_{\tau, s}^{+}$and $R e_{\theta}$, see table 7 . Error bars with a relative 


\section{T. Knopp and others}

magnitude of $5 \%$ for $u^{+}$and $6 \%$ for $y^{+}$are included, based on the relative uncertainty in $u_{\tau}$ of $5 \%$ and in $v$ of $1 \%$. In the inner layer, the reduction of the log-law region and the emerging of the sqrt law above the log law are similar for the two flows. One should be cautious, however, due to the uncertainty of the $u_{\tau}$-values reported in Coles \& Hirst (1969) which rely on the CCM with $\kappa=0.41$ and $B=5.0$, see $\S 4.4$, and deviations of the $u^{+}$-profiles within the error bounds are possible. The comparable inner layer behaviour indicates the influence of the local effects and the rapid response of the inner layer. The differences in the outer layer are due to the different flow history, leading to larger values $H_{12}=1.86$ and $H_{12}=1.98$ for the flow by Schubauer \& Klebanoff. For comparison, $H_{12}=2.0$ was found for the equilibrium flow by Skare \& Krogstad (1994) at almost the same $\Delta p_{s}^{+}, \operatorname{Re}_{\theta}$ and $\beta_{R C}$, but at a much smaller $\Delta u_{\tau, s}^{+}$. These findings are in agreement with the result by Bobke et al. (2017), that $\beta_{R C}$ alone is not sufficient to determine the mean-velocity profile at APG. A more detailed view reveals that the extent of the sqrt law is a little smaller for the present flow than for the flow by Schubauer \& Klebanoff. This is an indication that the outer part of the inner layer is probably affected by historical effects of the outer layer.

To summarise, the observed rapid recovery of the log-law region downstream of the end of curvature is in agreement with the results for the eddy turnover length $\delta_{t . o}$. in figure 5(c), indicating that the inner layer relaxes rapidly, albeit not instantaneously. The mean velocity in the outer layer was found to be influenced by history effects due to streamwise changing pressure gradient and convex curvature in conjunction with the slow response of the outer layer indicated by the large values for $\delta_{t . o .}$. The history effects were found to affect the outer part of the inner layer, causing the observed reduced extent of the sqrt-law region compared to the region occupied by the log law at ZPG.

\section{Conclusions}

The goal of the analysis was to determine the resilience of the log law for the mean velocity, the possible change of the von Kármán constant $\kappa$ and the appearance of a square-root law above the log law at significant APGs. We observed that, while the log law in the mean-velocity profile is a robust feature at APG, this region is thinner than its ZPG counterpart, and its slope is altered. A square-root law emerges above the log law in a large part of the region the log law occupies at ZPG. The square-root law was detected by a plateau of the mean-velocity slope diagnostic function. As the plateau was found to have only a moderate extent even for the large Reynolds number $\operatorname{Re}_{\theta}=57000$, we infer that the square-root-law region appears only at sufficiently large Reynolds numbers. This structure of the mean velocity profile confirms the proposal by Perry et al. (1966).

It was found that, when the pressure gradient parameter $\Delta p_{s}^{+}$increases from zero to 0.018 , the value of $\kappa$ decreases from $0.395 \pm 0.013$ to $0.370 \pm 0.017$. For comparison, the model by Nickels (2004) predicts a reduction from $\kappa=0.390$ for $\Delta p_{s}^{+}=0$ to $\kappa=0.365$ for $\Delta p_{s}^{+}=0.018$. Using the value for $u_{\tau}$ from the Clauser chart was shown to give erroneously smaller values for $\kappa$ than if a direct method for $u_{\tau}$ was used. The reduction of $\kappa$ could not be demonstrated to be significant due to the measurement uncertainty, and because of possible history effects of the upstream located region of streamwise changing pressure gradient and convex wall curvature. The major contribution to the overall uncertainty of $\kappa$ stems from the wall shear stress. Complementary to OFI, two indirect methods for the wall shear stress were found to be promising, viz., using a least-squares fit to the mean-velocity profile by Nickels (2004) in the region $y^{+}<20$, and the relation for the total shear stress derived from the integral form of the mean momentum balance, see Volino \& Schultz (2018). 
Finally the findings are put in context with other turbulent boundary layer flows at APG. In the inner layer, the reduction of the log-law region and the emerging of the sqrt law above the log law were found to be similar to the flow by Schubauer \& Klebanoff in Coles $\&$ Hirst (1969). Both data are at a similar $\operatorname{Re}_{\theta}$ and $\beta_{R C}$, and at almost the same values for $\Delta p_{s}^{+}$and wall shear stress gradient parameter $\Delta u_{\tau, s}^{+}$. This indicates the rapid response of the inner layer and the role of local effects on the inner layer. Significant historical effects were observed for the outer layer, and these were found to affect the outer part of the inner layer and the extent of the sqrt-law region. As most turbulent boundary layer flows of practical relevance are streamwise evolving flows, future research on non-equilibrium and history effects seems to be highly appreciated.

Acknowledgements. The authors gratefully acknowledge the assistance of M. Bitter, F. Eich, R. Hain, S. Scharnowski, J. Agocs and R. Geisler for the wind-tunnel experiment. The authors are very grateful to the partners of the International Collaboration on Experimental Turbulence (ICET) and in particular to S.C.C. Bailey and to I. Marusic for kindly providing their data. Finally the very valuable reviewer comments are gratefully acknowledged.

Funding. The experiment was funded by DFG (Grant KA 1808/14-1 \& SCHR 1165/3-1) and by the DLR Institute of Aerodynamics and Flow Technology.

Declaration of interests. The authors report no conflict of interest.

Author ORCIDs.

(D) Tobias Knopp https://orcid.org/0000-0002-3161-5353;

(1) N. Reuther https://orcid.org/0000-0003-3399-8367;

(D) E. Schülein https://orcid.org/0000-0002-1125-8504.

\section{Appendix A. Theoretical relations}

\section{A.1. Boundary layer theory and scaling to viscous units}

Consider a two-dimensional, incompressible turbulent boundary layer flow. A wall-fitted local coordinate system with streamwise wall-parallel direction $s$, wall-normal direction $y$ and mean-velocity components $U$ (streamwise wall parallel) and $V$ (wall normal) is assumed together with the following boundary layer equation for $U$

$$
v \frac{\partial^{2} U}{\partial y^{2}}-\frac{\partial \overline{u^{\prime} v^{\prime}}}{\partial y}=\frac{1}{\rho} \frac{\mathrm{d} P_{w}}{\mathrm{~d} s}+U \frac{\partial U}{\partial s}+V \frac{\partial U}{\partial y}+\frac{\partial}{\partial s}\left(\overline{u^{\prime 2}}-\overline{v^{\prime 2}}\right) .
$$

Here, the relation $P(s, y)=P_{w}(s)-\overline{v^{\prime 2}}(s, y)$ is used, which can be obtained by integration of the boundary layer equation for $V$, see Hinze (1975). Then integration of (A1) from the wall to the wall distance $y$ gives the following relation for the total shear stress $\tau$

$$
\begin{gathered}
\frac{\tau}{\rho} \equiv v \frac{\partial U}{\partial y}-\overline{u^{\prime} v^{\prime}}=\frac{\tau_{w}}{\rho}+\frac{1}{\rho} \frac{\mathrm{d} P_{\mathrm{w}}}{\mathrm{d} s} y+\int_{0}^{y} U \frac{\partial U}{\partial s} \mathrm{~d} \tilde{y} \\
+\int_{0}^{y} V \frac{\partial U}{\partial \tilde{y}} \mathrm{~d} \tilde{y}+\int_{0}^{y} \frac{\partial}{\partial s}\left(\overline{u^{\prime 2}}-\overline{v^{\prime 2}}\right) \mathrm{d} \tilde{y},
\end{gathered}
$$

where $\tau_{w}$ denotes the wall shear stress. The classical scaling to inner viscous units reads

$$
u^{+}=\frac{U}{u_{\tau}}, \quad y^{+}=\frac{y u_{\tau}}{v}, \quad \tau^{+}=\frac{\tau}{\tau_{w}}, \quad u_{\tau}=\sqrt{\frac{\tau_{w}}{\rho}}, \quad \Delta p^{+}=\frac{v}{\rho u_{\tau}^{3}} \frac{\mathrm{d} P_{w}}{\mathrm{~d} s} .
$$

A second classical scaling based on the so-called pressure-viscosity velocity $u_{p}=$ $v / \rho\left|\mathrm{d} P_{w} / \mathrm{d} s\right|$ by Stratford (1959) and Mellor (1966) is not used in this work, since $u_{\tau}>10 u_{p}$ in the region of interest of the present work. 


\section{T. Knopp and others}

\section{A.2. Relations for the mean velocity at APGs}

\section{A.2.1. A model for the viscous sublayer thickness by T. Nickels}

Nickels (2004) proposes an analytical model for the mean velocity in boundary layers subjected to mild and moderately strong pressure gradients, which can be favourable and adverse. The viscous sublayer solution $U_{v i s}^{+}=U_{v i s}^{+}\left(y^{+} ; \Delta p_{s}^{+}, y_{c}^{+}\right)$is given by

$U_{v i s}^{+}=y_{c}^{+}\left[1-\left[1+2 \frac{y^{+}}{y_{c}^{+}}+\frac{1}{2}\left(3-\Delta p_{s}^{+} y_{c}^{+}\right)\left(\frac{y^{+}}{y_{c}^{+}}\right)^{2}-\frac{3}{2} \Delta p_{s}^{+} y_{c}^{+}\left(\frac{y^{+}}{y_{c}^{+}}\right)^{3}\right] \mathrm{e}^{-3\left(y^{+} / y_{c}^{+}\right)}\right]$,

which depends on the local value of $\Delta p_{s}^{+}$and a parameter $y_{c}^{+}$, which is associated with the thickness of the viscous sublayer. Then $y_{c}$ is determined by a stability argument using a critical value $R_{c}$ of a suitably defined local Reynolds number

$$
R e_{c}=\frac{u_{T} y_{c}}{v}, \quad u_{T}=\left(\frac{\left.\tau\right|_{y=y_{c}}}{\rho}\right)^{1 / 2},\left.\quad \tau^{+}\right|_{y=y_{c}}=1+\Delta p_{s}^{+} y_{c}^{+} .
$$

Here, $y_{c}$ is the critical value of $y$ above which the viscous sublayer becomes unstable. The assumption that $\operatorname{Re}_{c}$ has a universal value for all wall-bounded flows implies that

$$
\Delta p_{s}^{+}\left(y_{c}^{+}\right)^{3}+\left(y_{c}^{+}\right)^{2}-\operatorname{Re}_{c}^{2}=0,
$$

where $R e_{c}$ is the only free parameter and is chosen from ZPG data to $R e_{c}=12$. The physically relevant solution for $y_{c}$ is the smallest positive root of (A6).

\section{A.2.2. A model for the von Kármán constant $\kappa$ by T. Nickels}

Regarding the von Kármán constant $\kappa$, Nickels (2004) argues that two relations to characterise the vorticity scale in the inner layer should be equal. The first relation is based on the velocity scale $u_{\tau}$ and the second is based on $u_{T}$. This leads to

$$
\frac{\partial U}{\partial y}=\frac{u_{T}}{\kappa_{0} y}=\frac{u_{\tau}}{\kappa y} \Leftrightarrow \frac{\kappa}{\kappa_{0}}=\frac{u_{\tau}}{u_{T}} \Leftrightarrow \frac{\kappa}{\kappa_{0}}=\sqrt{\frac{1}{1+\Delta p_{s}^{+} y_{c}^{+}}}
$$

where $\kappa_{0}$ is the value of $\kappa$ for the ZPG case, for which $\kappa_{0}=0.39$ is chosen.

\section{A.2.3. Mean-velocity slope diagnostic functions}

The mean-velocity slope diagnostic function is a method to assess the functional behaviour of the mean-velocity gradient, see e.g. Österlund et al. (2000) for ZPG flows. The diagnostic functions for log law and sqrt law (1.3) are

$$
\xi_{l o g}^{-1}=y^{+}\left(\frac{\mathrm{d} u^{+}}{\mathrm{d} y^{+}}\right)_{d a t a}, \quad \xi_{s q r t}^{-1}=\frac{y^{+}}{\sqrt{1+\Delta p_{s}^{+} y^{+}}}\left(\frac{\mathrm{d} u^{+}}{\mathrm{d} y^{+}}\right)_{d a t a} .
$$

A plateau region of the slope diagnostic function supports the assumed behaviour of $u^{+}\left(y^{+}\right)$. At ZPG, a plateau can be seen only for $R e_{\theta} \gtrsim 6000$, see Österlund et al. (2000). The overlap of inner and outer layer becomes sufficiently thick in terms of $y^{+}$only for asymptotically high $R e$. At APG, we suppose that at least $\operatorname{Re}_{\theta}>10000$ is needed to observe an asymptotic behaviour with a sufficiently thick overlap region. This was found in a study of the database by Coles \& Hirst (1969) in Knopp (2016). 


$\begin{array}{lcccccc}\begin{array}{l}U_{\infty} \\ \text { in } \mathrm{m} \mathrm{s}^{-1}\end{array} & \begin{array}{c}\text { Meas. } \\ \text { techn. }\end{array} & \begin{array}{c}T_{\infty} \\ \text { in K }\end{array} & \begin{array}{c}p_{\infty} \\ \text { in Pa }\end{array} & \begin{array}{c}\rho_{\infty} \\ \text { in } \mathrm{kg} \mathrm{m}^{-3}\end{array} & \begin{array}{c}\mu_{\infty} \times 10^{-5} \\ \text { in } \mathrm{Ns} \mathrm{m}^{-2}\end{array} & \begin{array}{c}v_{\infty} \times 10^{-5} \\ \text { in m }^{2} \mathrm{~s}^{-1}\end{array} \\ 23 & \text { 2D2C PIV } & 285.43 & 95010 & 1.1600 & 1.7984 & 1.5503 \\ 23 & \text { 2D- } \mu \text { PTV } & 284.1 & 94992 & 1.1648 & 1.7918 & 1.5383 \\ 23 & \text { OFI } & 296.76 & 95006 & 1.1132 & 1.8199 & 1.6349 \\ 36 & \text { 2D2C PIV } & 284.58 & 94415 & 1.1558 & 1.7942 & 1.5524 \\ 36 & \text { three-dimensional LPT STB } & 284.58 & 94415 & 1.1564 & 1.7942 & 1.5515\end{array}$

Table 8 . Summary of flow conditions in the wind tunnel.

\section{Appendix B. Flow conditions during the wind-tunnel measurements}

The flow conditions for the different measurement campaigns are summarised in table 8 . The reference pressure $p_{\infty}$ was measured downstream of the contour model near the exit of the test section.

\section{Appendix C. Determination of the boundary layer thickness}

As a boundary layer at APG exists below irrotational free streams with non-zero wall-normal variations, Coleman et al. (2018) proposed $\tilde{\delta}_{99}$ based on the velocity $\tilde{U}$

$$
\tilde{U}(s, y)=-\int_{0}^{y} \bar{\omega}_{z}\left(s, y^{\prime}\right) \mathrm{d} y^{\prime}, \quad \bar{\omega}_{z}=\frac{\partial V}{\partial s}-\frac{\partial U}{\partial y}
$$

In the APG region on the inclined flat plate, the difference between $\delta_{99}$ and $\tilde{\delta}_{99}$ is $1 \%$. An advantage of $\tilde{\delta}_{99}$ is that $\tilde{U}$ shows a clearer region of $\partial \tilde{U} / \partial y=0$.

In the convex curvature region the pressure increases with distance to the wall, and there is a corresponding decrease in velocity in the inviscid flow. Following Patel \& Sotiropoulos (1997), the boundary layer thickness needs to be defined using the potential flow velocity distribution $U_{p}=U_{p w}(1-K y)$, where the curvature $K=1 / R_{c}$ is positive for a convex wall, and $U_{p w}$ is the hypothetical potential flow velocity at the wall, which is found from $c_{p}$ using the Bernoulli equation. Figure 3 shows that $U(y)$ approaches $U_{p}(y)$ at $x=9.42 \mathrm{~m}$ in the region of approximately constant surface curvature. The wall distance, where $U$ reaches $0.99 U_{p}$, is $\delta=0.144 \mathrm{~m}$ compared to $\delta_{99}=0.131 \mathrm{~m}$.

Moreover, the two criteria proposed in Vinuesa et al. (2016) were studied, i.e. the wall distance where (i) $u^{\prime} / U=0.02$ and (ii) where $u^{\prime} /\left(U \sqrt{H_{12}}\right)=0.02$. The former gives better agreement with $\delta_{99}$ and $\tilde{\delta}_{99}$. It shows slightly smaller values than $\delta_{99}$. The deviation is below $5 \%$ in the ZPG region and $3.7 \%$ on the flat wall in the APG region. Finally, the results for the TNTI by Reuther \& Kähler (2018) are used, which were evaluated using an intermittency factor of $\gamma=0.3$, see Reuther (2019). The deviation of $\delta_{99}$ from these values is smaller than $2.5 \%$ in the ZPG region and $4 \%$ in the curvature region. In the APG region, the values are up to $7.5 \%$ larger than $\delta_{99}$.

\section{Appendix D. Mean-velocity slope diagnostic function for the log-law}

The mean-velocity slope diagnostic function for the three-dimensional LPT data for the case $U_{\infty}=36 \mathrm{~m} \mathrm{~s}^{-1}$ is shown in figure $16(a)$. The smoothing method described in $\S 4.6$ was applied to the data. An approximative plateau is found in the region $84<y^{+}<152$. 


\section{T. Knopp and others}
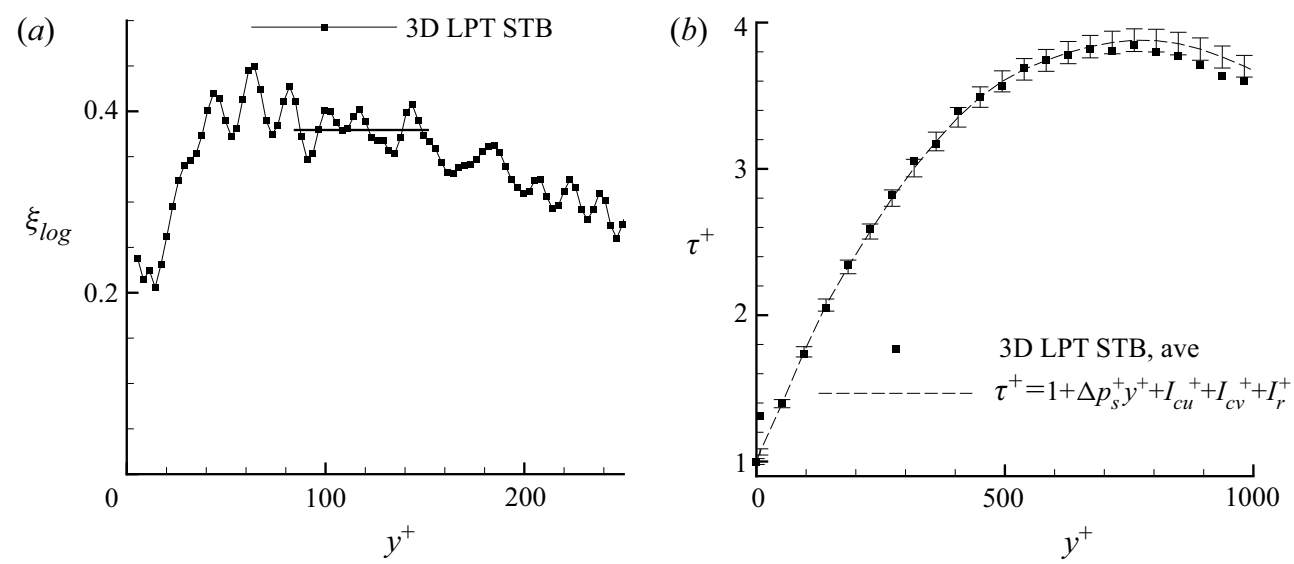

Figure 16. (a) Mean-velocity slope diagnostic function for the log law for $U_{\infty}=36 \mathrm{~m} \mathrm{~s}^{-1}$. (b) Determination of $u_{\tau}$ by a least-square fit of the measured total shear stress (denoted by 3D LPT STB, ave) and the remaining terms of the integral momentum balance (A2), see (3) in Volino \& Schultz (2018). For the nomenclature see $(\mathrm{E} 1 a-c)$.

\section{Appendix E. Method to determine the friction velocity from the mean momentum balance}

In figure $16(b) u_{\tau}$ is determined by a least square fit of the total shear stress and the remaining terms of the integral mean momentum balance on the right-hand side of (A2), after scaling both sides to viscous units. Therein a $2 \%$ error bar for the right-hand side of (A2) is shown. For abbreviation in figure $16(b)$, we use the notation

$$
I_{c u}(y)=\int_{0}^{y} U \frac{\partial U}{\partial s} \mathrm{~d} \tilde{y}, \quad I_{c v}(y)=\int_{0}^{y} V \frac{\partial U}{\partial \tilde{y}} \mathrm{~d} \tilde{y}, \quad I_{r}(y)=\int_{0}^{y} \frac{\partial}{\partial s}\left(\overline{u^{\prime 2}}-\overline{v^{\prime 2}}\right) \mathrm{d} \tilde{y},
$$

to denote the integrated convective term and the Reynolds normal stress term. This approach corresponds to the use of (3) in Volino \& Schultz (2018). The estimate of an uncertainty of $5 \%$ for $u_{\tau}$ is based on the uncertainty of $3.5 \%$ for $\rho^{-1} \mathrm{~d} P_{w} / \mathrm{d} s$ and of $1.5 \%$ for the remaining terms of $(\mathrm{E} 1 a-c)$ for the three-dimensional LPT data.

\section{E.1. A model for the total shear stress}

An analytical model for the total shear stress by Coles (1956) and Perry (1966) is based on the ansatz for the mean-velocity profile in the inner region $U(s, y)=u_{\tau}(s) f\left(y^{+}(s, y)\right)$ and $y^{+}(s, y)=u_{\tau}(s) y / v$ to model $(\mathrm{E} 1 a-c)$. By neglecting $I_{r}^{+}\left(y^{+}\right)$this gives

$$
\tau^{+}\left(y^{+}\right)=1+\Delta p_{s}^{+} y^{+}+\Delta u_{\tau, s}^{+} I_{u}^{+}, \quad \Delta u_{\tau, s}^{+}=\frac{v}{u_{\tau}^{2}} \frac{\mathrm{d} u_{\tau}}{\mathrm{d} s} \quad I_{u}^{+}=\int_{0}^{y^{+}} f^{2} \mathrm{~d} \tilde{y}^{+} . \quad(\mathrm{E} 2 a-c)
$$

The local effects on the total shear stress in the inner layer involve $\Delta p_{s}^{+}$and the wall shear stress gradient parameter $\Delta u_{\tau, s}^{+}$. Note that an extension of the ansatz (E2a-c) using $f=f\left(y^{+}(s, y), \Delta p_{s}^{+}(s)\right)$ accounts for higher-order effects on $\tau^{+}$, involving an additional parameter based on $\mathrm{d}^{2} P / \mathrm{d} s^{2}$, see Knopp et al. (2015). 
Sources of uncertainties

No reliable data points below $y^{+} \leqslant 1.9$

Spreading in $U(y)$ due to not enough samples

Uncertainty in the wall position $\left(\Delta y^{+}=0.3\right)$

Possible measurement error of data points $U(y)$ for $y^{+}<2.6\left(\epsilon_{U}\right.$ of $\left.2 \%\right)$

Uncertainty due to an estimated uncertainty in $v$ of $1 \%$

Estimated total uncertainty
Uncertainty of $u_{\tau}$
$1.6 \%$
$0.5 \%$
$0.5 \%$
$1.3 \%$
$0.5 \%$
$4.4 \%$

Table 9. Uncertainty quantification for $u_{\tau}$ for the least-squares fit to the viscous sublayer profile $u^{+}=y^{+}$in the region $y^{+} \in[2 ; 4.6]$ for the $\mu \mathrm{PTV}$ data at $U_{\infty}=23 \mathrm{~m} \mathrm{~s}^{-1}$.

Sources of uncertainties

Variation of lower and upper bound for the fit $y^{+} \in[4 \pm 2,17 \pm 3]$

Uncertainty in the wall position $\left(\Delta y^{+}=0.3\right)$

Uncertainty due to an estimated uncertainty in $v$ of $1 \%$

Additional unknown systematic uncertainty due to the fit function (A4)

Estimated total uncertainty
Uncertainty of $u_{\tau}$

$$
\begin{gathered}
<0.1 \% \\
1.0 \% \\
0.3 \% \\
2.5 \% \\
3.8 \%
\end{gathered}
$$

Table 10. Uncertainty quantification for $u_{\tau}$ for the least-squares fit to the profile by Nickels (2004) in the region $y^{+} \in[2 ; 20]$ for the $\mu \mathrm{PTV}$ data at $U_{\infty}=23 \mathrm{~m} \mathrm{~s}^{-1}$.

\section{Appendix F. Uncertainty analysis}

In this section the uncertainty analysis for the different methods to determine the friction velocity $u_{\tau}$, and for the evaluation of $\kappa$ is presented.

\section{F.1. Determination of the friction velocity}

For the OFI results at $U_{\infty}=23 \mathrm{~m} \mathrm{~s}^{-1}$, the uncertainty in $u_{\tau}$ is estimated to be $2.0 \%$. This estimate is based on the work by Thibault \& Poitras (2017). For the current application case (oil viscosity is calibrated up to a standard uncertainty of $1 \%$, re-projection of the interference images onto the model surface with an accuracy on the local scale of better than $1 \%$, improving accuracy by statistical and local averaging of multiple independent measurements, etc.) the standard uncertainty of OFI for determining $u_{\tau}$ is expected to be better than $\pm 2 \%$ (with a $95 \%$ confidence level). For comparison Harun et al. (2013) report an uncertainty of $1 \%$.

For the method to determine $u_{\tau}$ by a least-squares fit of the data to the relation $u^{+}=y^{+}$ in the region $y^{+} \in[2 \pm 0.1 ; 4.6 \pm 0.6]$, the sources of uncertainties are listed in table 9 . For the overall uncertainty for $u_{\tau}$ the estimate is $4.4 \%$.

For the method to determine $u_{\tau}$ using a least-squares fit of the data to the profile by Nickels (2004) in the region $y^{+} \in[2 ; 20]$, the sources of uncertainties are listed in table 10 . The uncertainty due to the assumed profile by Nickels (2004) was estimated by a statistical evaluation and comparison with the data by Nagano et al. (1991), Manhart \& Friedrich (2002) and Coleman et al. (2018) in the interval given above, and is denoted by additional unknown systematic uncertainty. The overall uncertainty for $u_{\tau}$ is $3.8 \%$.

The sources of uncertainties for the method to determine $u_{\tau}$ using a fit to the profile by Nickels (2004) in the region $y^{+} \in[5 ; 20]$ for the three-dimensional LPT data at $U_{\infty}=$ $36 \mathrm{~m} \mathrm{~s}^{-1}$ are listed in table 11 . The overall uncertainty for $u_{\tau}$ is estimated to be $4.0 \%$. 
Sources of uncertainties

Variation of upper bound for the fit $y^{+} \in[5,16.5 \pm 3.5]$

Uncertainty in the wall position $\left(\Delta y^{+}=0.3\right)$

Uncertainty due to an estimated uncertainty in $v$ of $1 \%$

Additional unknown systematic uncertainty due to the fit function (A4)

Estimated total uncertainty
Uncertainty of $u_{\tau}$

$0.3 \%$

$0.8 \%$

$0.4 \%$

$2.5 \%$

$4.0 \%$

Table 11. Uncertainty quantification for $u_{\tau}$ for the fit to the profile by Nickels (2004) in the region $y^{+} \in[5 ; 20]$ for the three-dimensional LPT data for $U_{\infty}=36 \mathrm{~m} \mathrm{~s}^{-1}$.

Sources of uncertainties

Variation of $y_{\log , \min }^{+}, y_{\log , \max }^{+}$within the specified limits

Uncertainty in the wall position $\left(\Delta y^{+}=0.3\right)$

Uncertainty due to an estimated uncertainty in $v$ of $1 \%$

Uncertainty due to $2 \%$ uncertainty in $u_{\tau}$ for OFI

Uncertainty due to $4.4 \%$ uncertainty in $u_{\tau}$ for fit to $u^{+}=y^{+}$

Uncertainty due to $3.8 \%$ uncertainty in $u_{\tau}$ for fit to profile by Nickels (2004)

Uncertainty due to $6.1 \%$ uncertainty in $u_{\tau}$ for CCM
Uncertainty of $\kappa$
$2.0 \%$
$0.25 \%$
$0.25 \%$
$2.0 \%$
$4.4 \%$
$3.8 \%$
$6.1 \%$

Table 12. Sources of uncertainties for the determination of $\kappa$ and uncertainty quantification for the $\mu$ PTV data for $U_{\infty}=23 \mathrm{~m} \mathrm{~s}^{-1}$.

Sources of uncertainties

Variation of $y_{\log , \min }^{+}, y_{\log , \max }^{+}$within the specified limits

Uncertainty in the wall position $\left(\Delta y^{+}=0.3\right)$

Uncertainty due to an estimated uncertainty in $v$ of $1 \%$

Uncertainty due to $4.0 \%$ uncertainty in $u_{\tau}$ for fit to profile by Nickels

Uncertainty due to $5.6 \%$ uncertainty in $u_{\tau}$ for CCM

Uncertainty due to $5.6 \%$ uncertainty in $u_{\tau}$ for corrected CCM
Uncertainty of $\kappa$

$0.9 \%$

$0.3 \%$

$<0.1 \%$

$4.0 \%$

$5.6 \%$

$5.6 \%$

Table 13. Sources of uncertainties for the determination of $\kappa$ and uncertainty quantification for the three-dimensional LPT data for $U_{\infty}=36 \mathrm{~m} \mathrm{~s}^{-1}$.

\section{F.2. Determination of the von Kármán constant $\kappa$}

The sources for uncertainty for $\kappa$ are summarised in table 12 for $U_{\infty}=23 \mathrm{~m} \mathrm{~s}^{-1}$ and in table 13 for $U_{\infty}=36 \mathrm{~m} \mathrm{~s}^{-1}$. The uncertainty due to the choice of the log-law region is computed by a statistical variation of $y_{\log , \min }^{+}$and $y_{\log , \max }^{+}$, motivated by the Monte Carlo-based error analysis in Bailey et al. (2014). For $U_{\infty}=23 \mathrm{~m} \mathrm{~s}^{-1}$ the lower bound was $y_{\log , \min }^{+} \in[82 ; 92]$ and the upper bound was $y_{\log , \max }^{+} \in[126 ; 138]$. For $U_{\infty}=36 \mathrm{~m} \mathrm{~s}^{-1}$, $y_{\log , \text { min }}^{+} \in[82 ; 90]$ and $y_{\log , \max }^{+} \in[142 ; 150]$ was used. The overall uncertainties for $\kappa$ are given in the last column in table 4 for $U_{\infty}=23 \mathrm{~m} \mathrm{~s}^{-1}$ and in table 5 for $U_{\infty}=36 \mathrm{~m} \mathrm{~s}^{-1}$.

\section{F.3. Clauser chart method in the APG region}

For the uncertainty of the CCM, Monty et al. (2011) report an uncertainty for $u_{\tau}$ of $5 \%$ for $\beta_{R C} \geqslant 2$. For the Preston tube, Patel (1965) gives an accuracy within $6 \%$ for $\Delta p_{s}^{+}<0.015$, see also Brown \& Joubert (1969). For the present flow, the deviation of $u_{\tau}$ determined by 
Sources of uncertainties

Variation of $y_{\log , \min }^{+}, y_{\log , \max }^{+}$within the specified limits

Variation of log-law coefficients $(\kappa=0.41, B=5.0$ vs $\kappa=0.384, B=4.17$ )

Uncertainty of the wall position $\left(\Delta y^{+}=0.3\right)$

Uncertainty due to an estimated uncertainty in $v$ of $1 \%$

Systematic error of CCM compared to OFI and fit to $u^{+}=y^{+}$

Estimated total uncertainty
Uncertainty of $u_{\tau}$
$0.2 \%$
$0.3 \%$
$0.4 \%$
$0.2 \%$
$5.0 \%$
$6.1 \%$

Table 14. Sources of uncertainties for the determination of $u_{\tau}$ using the CCM and uncertainty quantification for the $\mu \mathrm{PTV}$ data at $U_{\infty}=23 \mathrm{~m} \mathrm{~s}^{-1}$.

Sources of uncertainties

Variation of $y_{\log , \min }^{+}, y_{\log , \max }^{+}$within the specified limits

Variation of log-law coefficients $(\kappa=0.41, B=5.0$ vs $\kappa=0.384, B=4.17$ )

Uncertainty in the wall position $\left(\Delta y^{+}=0.3\right)$

Uncertainty due to an estimated uncertainty in $v$ of $1 \%$

Systematic error of CCM compared to OFI and fit to $u^{+}=y^{+}$

Estimated total uncertainty
Uncertainty of $u_{\tau}$

$$
\begin{gathered}
0.15 \% \\
0.2 \% \\
0.05 \% \\
0.15 \% \\
5.0 \% \\
5.6 \%
\end{gathered}
$$

Table 15. Sources of uncertainties for the determination of $u_{\tau}$ using the CCM and uncertainty quantification for the three-dimensional LPT data at $U_{\infty}=36 \mathrm{~m} \mathrm{~s}^{-1}$.

the CCM is smaller than $5 \%$ compared to the value for $u_{\tau}$ from the most reliable method. Therefore, a systematic error of $5 \%$ for the CCM is assumed. Moreover, the additional sources for uncertainties need to be taken into account. The sensitivity with respect to the interval $\left[y_{\log , \min }^{+}, y_{\log , \text { max }}^{+}\right]$is estimated by a statistical variation of the lower and upper bounds. This gives a relative uncertainty based on the $\pm 2 \sigma$-interval of $0.65 \%$ for $U_{\infty}=$ $23 \mathrm{~m} \mathrm{~s}^{-1}$ and a value below $0.1 \%$ for $U_{\infty}=36 \mathrm{~m} \mathrm{~s}^{-1}$. The results are summarised in table 14 for $U_{\infty}=23 \mathrm{~m} \mathrm{~s}^{-1}$ and in table 15 for $U_{\infty}=36 \mathrm{~m} \mathrm{~s}^{-1}$.

\section{Appendix G. Empirical correction method for the Clauser chart}

An empirical method was used to correct the standard CCM for $u_{\tau}$ for the three-dimensional LPT data for $U_{\infty}=36 \mathrm{~m} \mathrm{~s}^{-1}$. It is based the observation found in this work and by Monty et al. (2011), that the value for $u_{\tau}$ by the CCM is lower than the value determined by a direct method. For simplicity assume that the correction is a linear function of $\Delta p_{s}^{+}$neglecting higher-order terms. Putting this together, we use the empirical correction

$$
u_{\tau, C C M+\operatorname{corr}, 36}=u_{\tau, C C M, 36}\left(1+\frac{u_{\tau, v i s, 23}-u_{\tau, C C M, 23}}{u_{\tau, C C M, 23}} \frac{\Delta p_{s, 36}^{+}}{\Delta p_{s, 23}^{+}}\right),
$$

where an additional subscript is for the reference velocity, yielding $u_{\tau}=0.7885 \mathrm{~m} \mathrm{~s}^{-1}$.

\section{REFERENCES}

AfZAL, N. 1982 Fully developed turbulent flow in a pipe. An intermediate layer. Arch. Appl. Mech. 52, 355-377. 


\section{T. Knopp and others}

AfZAL, N. 2008 Turbulent boundary layer with negligible wall stress. Trans. ASME J. Fluids Engng 130, 051205 .

Alving, A.E. \& Fernholz, H.H. 1995 Mean-velocity scaling in and around a mild, turbulent separation bubble. Phys. Fluids 7, 1956-1969.

Alving, A.E., Smits, A.J. \& WATMUfF, J.H. 1990 Turbulent boundary layer relaxation from convex curvature. J. Fluid Mech. 211, 529-556.

Atkinson, C., Buchner, A.J., Eisfelder, M., Kitsios, V. \& Soria, J. 2016 Time-resolved PIV measurements of a self-similar adverse pressure gradient turbulent boundary layer. In 18th International Symposium on the Application of Laser and Imaging Techniques to Fluid Mechanics, Lisbon, Portugal, July 4-7, 2016 (ed. D.F.G. Durão, K. Hishida, E.K. Longmire, A.L. Moreira \& C. Tropea). Springer.

BAILEY, S.C.C., et al. 2013 Obtaining accurate mean velocity measurements in high Reynolds number turbulent boundary layers using pitot tubes. J. Fluid Mech. 715, 642-670.

Bailey, S.C.C., Vallikivi, M., Hultmark, M. \& Smits, A.J. 2014 Estimating the value of von Kármán's constant in turbulent pipe flow. J. Fluid Mech. 749, 79-98.

BANDYOPADHYAY, P. \& AHMED, A. 1993 Turbulent boundary layers subjected to multiple curvatures and pressure gradients. J. Fluid Mech. 246, 503-527.

Baskaran, V., Smits, A.J. \& Joubert, P.N. 1987 A turbulent flow over a curved hill. Part 1. Growth of an internal boundary layer. J. Fluid Mech. 182, 47-83.

Bobke, A., Vinuesa, R., ÖRlü, R. \& Schlatter, P. 2017 History effects and near equilibrium in adverse-pressure-gradient turbulent boundary layers. J. Fluid Mech. 820, 667-692.

BRADShaw, P. 1970 Turbulence. Springer.

Bross, M., FuCHS, T. \& KäHLER, C.J. 2019 Interaction of coherent flow structures in adverse pressure gradient turbulent boundary layers. J. Fluid Mech. 873, 287-321.

BRown, K.C. \& JouberT, P.N. 1969 The measurement of skin friction in turbulent boundary layers with adverse pressure gradients. J. Fluid Mech. 35, 737-757.

Chauhan, K.A., Monkewitz, P.A. \& Nagib, H.M. 2009 Criteria for assessing experiments in zero pressure gradient boundary layers. Fluid Dyn. Res. 41, 021404.

Coleman, G.N., Pirozzoli, S., QuAdrio, M. \& Spalart, P.R. 2017 Direct numerical simulation and theory of a wall-bounded flow with zero skin friction. Flow Turbul. Combust. 99, 553-564.

Coleman, G.N., RumSey, C.L. \& Spalart, P.R. 2018 Numerical study of turbulent separation bubbles with varying pressure gradient and Reynolds number. J. Fluid Mech. 847, 28-70.

ColEs, D. 1956 The law of the wake in the turbulent boundary layer. J. Fluid Mech. 1, 191-226.

COLES, D.E. \& HIRST, E.A. 1969 Computation of Turbulent Boundary Layers - 1968 AFOSR-IFP-Stanford Conference. Thermosciences Division, Department of Mechanical Engineering, Stanford University.

Dennis, D.J.C. \& Nickels, T.B. 2011 Experimental measurement of large-scale three-dimensional structures in a turbulent boundary layer. Part 2. Long structures. J. Fluid Mech. 673, 218-244.

Dixit, S.A. \& RAMESH, O.N. 2009 Determination of skin friction in strong pressure-gradient equilibrium and near-equilibrium turbulent boundary layers. Exp. Fluids 47, 1045-1058.

DURBIN, P.A. \& BELCHER, S.E. 1992 Scaling of adverse-pressure-gradient boundary layers. J. Fluid Mech. 238, 699-722.

Eich, F., DE Silva, C.M., MARusic, I. \& KäHLeR, C.J. 2020 Towards an improved spatial representation of a boundary layer from the attached eddy model. Phys. Rev. Fluids 5, 034601.

Galbraith, R.A., Sjolander, S. \& Head, M.R. 1977 Mixing length in the wall region of turbulent boundary layers. Aeronaut. Q. 28, 97-110.

GeORGE, W.K. \& CASTILLO, L. 1997 Zero-pressure gradient turbulent boundary layer. Appl. Mech. Rev. 50, 689-729.

GiLlis, J.C. \& Johnston, J.P. 1983 Turbulent boundary-layer flow and structure on a convex wall and its redevelopment on a flat wall. J. Fluid Mech. 135, 123-153.

Gillis, J.C., Johnston, J.P., KAYs, W.M. \& Moffat, R.J. 1980 Turbulent boundary layer on a convex curved surface. Tech. Rep. HMT-31, Department of Mechanical Engineering, Stanford University.

Gungor, A.G., Maciel, Y., Simens, M.P. \& Soria, J. 2016 Scaling and statistics of large-defect adverse pressure gradient turbulent boundary layer. Intl J. Heat Fluid Flow 59, 109-124.

Harun, Z., Monty, J.P., Mathis, R. \& Marusic, I. 2013 Pressure gradient effects on the large-scale structure of turbulent boundary layers. J. Fluid Mech. 715, 477-498.

HINZE, J.O. 1975 Turbulence. McGraw-Hill.

Jones, M.B., MARUSic, I. \& PeRry, E.E. 2001 Evolution and structure of sink-flow turbulent boundary layers. J. Fluid Mech. 428, 1-27.

KADER, B.A. \& YAGLOM, A.M. 1978 Similarity treatment of moving-equilibrium turbulent boundary layers in adverse pressure gradients. J. Fluid Mech. 89, 305-342. 


\section{Log law at adverse pressure gradient}

Kähler, C.J., Scharnowski, S. \& Cierpka, C. $2012 a$ On the resolution limit of digital particle image velocimetry. Exp. Fluids 52, 1629-1639.

KÄHLER, C.J., Scharnowski, S. \& CierpKa, C. $2012 b$ On the uncertainty of digital PIV and PTV near walls. Exp. Fluids 52, 1641-1656.

Kim, N. \& RHODE, D.L. 2000 Streamwise curvature effect of the incompressible turbulent mean velocity over curved surfaces. Trans. ASME J. Fluids Engng 122, 547-551.

KLEWICKI, J.C., FIFE, P. \& WEI, T. 2009 On the logarithmic mean profile. J. Fluid Mech. 638, 73-93.

KNOPP, T. 2016 A new wall-law for adverse pressure gradient flows and modification of $k-\omega$ type RANS turbulence models. AIAA Paper 2016-0588.

Knopp, T., Buchmann, N.A., Schanz, D., Eisfeld, B., Cierpka, C., Hain, R., Schröder, A. \& KÄHLER, C.J. 2015 Investigation of scaling laws in a turbulent boundary layer flow with adverse pressure gradient using PIV. J. Turbul. 16, 250-272.

Knopp, T., Schanz, D., Schröder, A., Buchmann, N.A., Cierpka, C., Hain, R. \& Kähler, C.J. $2014 a$ Experimental investigation of a turbulent boundary layer at adverse pressure gradient at $\operatorname{Re}_{\theta}$ up to 10000 using large-scale and long-range microscopic particle imaging. In WALLTURB Workshop: Progress in Wall Turbulence: Understanding and Modelling, 18-20 June 2014, Lille, France (ed. M. Stanislas, J. Jimenez \& I. Marusic), pp. 271-281. Springer.

Knopp, T., Schanz, D., Schröder, A., Dumitra, M., Hain, R. \& Kähler, C.J. 2014b Experimental investigation of the log-law for an adverse pressure gradient turbulent boundary layer flow at $\operatorname{Re}_{\theta}$ up to 10 000. Flow Turbul. Combust. 92, 451-471.

LEE, J.-H. \& SUNG, H.J. 2009 Structures in turbulent boundary layers subjected to adverse pressure gradients. J. Fluid Mech. 639, 101-131.

LONG, R.R. \& CHEN, T.C. 1981 Experimental evidence for the existence of the 'mesolayer' in turbulent systems. J. Fluid Mech. 105, 19-59.

MANHART, M. \& FRIEDRICH, R. 2002 DNS of a turbulent boundary layer with separation. Intl J. Heat Fluid Flow 23, 672-581.

Marusic, I., Chauhan, K.A., Kulandaivelu, V. \& Hutchins, N. 2015 Evolution of zero-pressure-gradient boundary layers from different tripping conditions. J. Fluid Mech. 783, 379-411.

Marusic, I., Monty, J.P., Hultmark, M. \& Smits, A.J. 2013 On the logarithmic region in wall turbulence. J. Fluid Mech. 716, R3.

MeLlor, G.L. 1966 The effects of pressure gradients on turbulent flow near a smooth wall. J. Fluid Mech. 24, 255-274.

Mellor, G.L. \& Gibson, D.M. 1966 Equilibrium turbulent boundary layers. J. Fluid Mech. 24, 225-253.

Monkewitz, P.A., Chauhan, K.A. \& Nagib, H.M. 2007 Comparison of mean flow similarity laws in zero pressure gradient turbulent boundary layers. Phys. Fluids 20, 105102.

Monkewitz, P.A., Chauhan, K.A. \& Nagib, H.M. 2008 Self-consistent high-Reynolds number asymptotics for ZPG turbulent boundary layers. Phys. Fluids 19, 115105.

MONTY, J.P., HARUN, Z. \& MARUSIC, I. 2011 A parametric study of adverse pressure gradient turbulent boundary layers. Intl J. Heat Fluid Flow 32, 575-585.

NAgAno, Y., TAgawa, M. \& Tsuji, T. 1991 Effects of adverse pressure gradients on mean flows and turbulence statistics in a boundary layer. In Eighth Symposium on Turbulent Shear Flows, Technical University of Munich, September 9-11, 1991 (ed. F. Durst, R. Friedrich, B.E. Launder, F.W. Schmidt, U. Schumann \& J.H. Whitelaw). Springer.

Nagib, H.M., Christophorou, C. \& Monkewitz, P.A. 2004 High Reynolds number turbulent boundary layers subjected to various pressure-gradient conditions. In IUTAM 2004: One Hundred Years of Boundary Layer Research, August 12-14, 2004, Göttingen, Germany (ed. G.E.A. Meier, K.R. Sreenivasan \& H.-J. Heinemann), pp. 383-394. Springer.

NiCKELS, T.B. 2004 Inner scaling for wall-bounded flows subject to large pressure gradients. J. Fluid Mech. $521,217-239$.

Novara, M., Schanz, D., Reuther, N., Kähler, C.J. \& Schröder, A. 2016 Lagrangian 3D particle tracking in high-speed flows: Shake-The-Box for multi-pulse systems. Exp. Fluids 57 (8), 1-20.

Österlund, J.M., Johansson, A.V., Nagib, H.M. \& Hites, M.H. 2000 A note on the overlap region in turbulent boundary layers. Phys. Fluids 12, 1-4.

Patel, V.C. 1965 Calibration of the preston tube and limitations on its use in pressure gradients. J. Fluid Mech. 23, 185-208.

Patel, V.C. \& Sotiropoulos, S. 1997 Longitudinal curvature effects in turbulent boundary layers. Prog. Aerosp. Sci. 33, 1-70.

PERRY, A.E. 1966 Turbulent boundary layers in decreasing adverse presssure gradients. J. Fluid Mech. 25, 481-506. 


\section{T. Knopp and others}

Perry, A.E., Bell, J.B. \& Joubert, P.N. 1966 Velocity and temperature profiles in adverse pressure gradient turbulent boundary layers. J. Fluid Mech. 25, 299-320.

RAMAPRIAN, B.R. \& ShIVAPRASAD, B.G. 1978 The structure of turbulent boundary layers along mildly curved surfaces. J. Fluid Mech. 85, 273-303.

REUTHER, N. 2019 Impact of the intermittent behavior on statistics in pressure gradient turbulent boundary layers. PhD thesis, Universität der Bundeswehr München, Fakultät für Luft- und Raumfahrttechnik, Institut für Strömungsmechanik und Aerodynamik.

Reuther, N. \& KÄHLER, C.J. 2018 Evaluation of large-scale turbulent/non-turbulent interface detection methods for wall-bounded flows. Exp. Fluids 59, 121.

Reuther, N. \& KÄHLER, C.J. 2020 Effect of the intermittency dynamics on single and multipoint statistics of turbulent boundary layers. J. Fluid Mech. 897, A11.

Reuther, N., Scharnowski, S., Hain, R., Schanz, D., Schröder, A. \& KÄhler, C.J. 2015 Experimental investigation of adverse pressure gradient turbulent boundary layers by means of large-scale PIV. In 11th International Symposium on Particle Image Velocimetry - PIV15, Santa Barbara, California, September 14-16, 2015.

Schatzman, D.M. \& Thomas, F.O. 2017 An experimental investigation of an unsteady adverse pressure gradient turbulent boundary layer: embedded shear layer scaling. J. Fluid Mech. 815, 592-642.

SCHLATtER, P. \& ÖRLÜ, R. 2010 Assessment of direct numerical simulation data of turbulent boundary layers. J. Fluid Mech. 659, 116-126.

Schülein, E., Reuther, N. \& KNOPP, T. 2017 Optical skin friction measurements in a turbulent boundary layer with pressure gradient. In New Results in Numerical and Experimental Fluid Mechanics. Contributions to the 20th STAB/DGLR Symposium Braunschweig, Germany 2016 (ed. A. Dillmann, G. Heller, C. Kämer, C. Wagner, S. Bansmer, R. Radespiel \& R. Semaan), pp. 95-104. Springer.

SCHULZE, S. 2012 Experimentelle Untersuchungen zur Wirbeldynamik am überziehenden Triebwerkseinlauf. PhD thesis, Universität der Bundeswehr München, Fakultät für Luft- und Raumfahrttechnik, Institut für Strömungsmechanik und Aerodynamik.

Sillero, J.A., JimeneZ, J. \& MOSER, R.D. 2013 One-point statistics for turbulent wall-bounded flows at Reynolds numbers up to $\delta^{+} \approx 2000$. Phys. Fluids 25, 105102.

SkARE, P.E. \& KROGSTAD, P.A. 1994 A turbulent equilibrium boundary layer near separation. J. Fluid Mech. 272, 319-348.

SPALART, P.R. 2010 The Law of the Wall. Indications from DNS, and Opinion. In Progress in Wall Turbulence: Understanding and Modelling. Proceedings of the WALLTURB International Workshop held in Lille, France, April 21-23, 2009 (ed. M. Stanislas, J. Jimenez \& I. Marusic), pp. 9-20. Springer.

SPALART, P.R. \& LEONARD, A. 1986 Direct numerical simulation of equilibrium turbulent boundary layers. In Proceedings of the 5th Symposium on Turbulent Shear Flows 5, Ithaca, USA, August 7-9, 1985 (ed. F.J. Durst, B.E. Launder, F.W. Schmidt \& J.H. Whitelaw), pp. 234-252. Springer.

STRATFORD, B.S. 1959 The prediction of separation of the turbulent boundary layer. J. Fluid Mech. 5, 1-16.

Thibault, R. \& Poitras, G.J. 2017 Uncertainty evaluation of friction velocity measurements by oil-film interferometry. Trans. ASME J. Fluids Engng 139, 051401.

Townsend, A.A. 1960 The development of turbulent boundary layers with negligible wall stress. J. Fluid Mech. 10, 143-155.

Townsend, A.A. 1961 Equilibrium layers and wall turbulence. J. Fluid Mech. 11, 97-120.

Vinuesa, R., BobKe, A., ÖRlü, R. \& SchlatTER, P. 2016 On determining characteristic length scales in pressure-gradient turbulent boundary layers. Phys. Fluids 28, 055101.

Volino, R.J. \& Schultz, M.P. 2018 Determination of wall shear stress from mean velocity and Reynolds shear stress profiles. Phys. Rev. Fluids 3, 034606.

Wei, T., Fife, P., Klewicki, J. \& McMurtry, P. 2005 Properties of the mean momentum balance in turbulent boundary layer, pipe and channel flows. J. Fluid Mech. 522, 303-327.

Zagarola, M.V. \& Smits, A.J. 1997 Scaling of the mean velocity profile for turbulent pipe flow. Phys. Rev. Lett. 78 (2), 239-242. 